\title{
A Hybrid High-Order method for passive transport in fractured porous media*
}

\author{
Florent Chave ${ }^{\dagger 1,2}$, Daniele A. Di Pietro ${ }^{\ddagger 1}$, and Luca Formaggia ${ }^{\S 2}$ \\ ${ }^{1}$ University of Montpellier, Institut Montpelliérain Alexander Grothendieck, 34095 Montpellier, France \\ ${ }^{2}$ Politecnico di Milano, MOX, 20133 Milano, Italy
}

September 8, 2018

\begin{abstract}
In this work, we propose a model for the passive transport of a solute in a fractured porous medium, for which we develop a Hybrid High-Order (HHO) space discretization. We consider, for the sake of simplicity, the case where the flow problem is fully decoupled from the transport problem. The novel transmission conditions in our model mimic at the discrete level the property that the advection terms do not contribute to the energy balance. This choice enables us to handle the case where the concentration of the solute jumps across the fracture. The HHO discretization hinges on a mixed formulation in the bulk region and on a primal formulation inside the fracture for the flow problem, and on a primal formulation both in the bulk region and inside the fracture for the transport problem. Relevant features of the method include the treatment of nonconforming discretizations of the fracture, as well as the support of arbitrary approximation orders on fairly general meshes.
\end{abstract}

Keywords: Hybrid High-Order methods, finite volume methods, finite element methods, fractured porous media, Darcy flow, miscible displacement, passive transport

MSC2010 classification: $76 \mathrm{~S} 05,65 \mathrm{~N} 08,65 \mathrm{~N} 30$,

\section{Introduction}

Over the last decades, the research on fluid flows in fractured porous media has received a great amount of attention because of its relevance in many areas of the geosciences, ranging from groundwater hydrology to hydrocarbon exploitation. Fractures in the subsurface are indeed ubiquitous, and can be caused by tectonic forces, changes of temperature, drying processes, by leaching in the plane of stratification, or by schistosity. Depending on the material that has accumulated within the fractures, they may act as conduits or barriers, and thus affect the flow patterns in a substantial way. For instance, it has been observed that fractures near boreholes tend to increase the productivity of wells during oil recovery. In the context of geological isolation of radioactive waste, the presence of

\footnotetext{
*The second author acknowledges the partial support of Agence Nationale de la Recherche grant HHOMM (ref. ANR15-CE40-0005-01). The third author acknowledges the support of INdaM-GNCS under the program Progetti 2017. The authors also acknowledge the support of the Vinci Programme of Université Franco Italienne.

†florent.chave@umontpellier.fr, corresponding author

\$daniele.di-pietro@umontpellier.fr

§luca.formaggia@polimi.it
}

Referee

$\# 1$,

typos 
fractures in the disposal areas due to, for example, tunnel excavation, can drastically accelerate the migration process of radionuclides.

A common feature of fractures in porous media is the variety of length scales. While the presence of smaller fractures may be accounted for by using homogenization or other upscaling techniques, fractures with larger extension have to be modelled explicitly, and there are several possible ways to incorporate their presence. Our focus is here on the approach developed in [29], where a reduced model for the flow in the fracture is obtained by an averaging process, and the fracture is treated as an interface inside the bulk region. The fracture is assumed to be filled of debris, so that the flow therein can still be modelled by Darcy's law. The problem is closed by interface conditions that relate the average and jump of the bulk pressure to the normal flux and pressure in the fracture. In [15] we have designed and analysed a Hybrid High-Order (HHO) method to discretize this model, and proved stability and order $O\left(h^{k+1}\right)$ convergence of the discretization error measured in an energy-like norm, with $h$ denoting the meshsize and $k \geq 0$ the polynomial degree. This method is based on a mixed formulation for the bulk coupled with a primal method for the fracture. This choice is motivated by the fact that the unknowns of the method are those that naturally appear in the coupling conditions (4), namely the normal component of the bulk flux and the fracture pressure. For a review of other formulations, we refer the reader to [24] for a review of different possible formulations. Concerning the equivalence of mixed and primal HHO methods, see $[1,12]$. Several other discretization schemes have been proposed for this type of models; see, e.g., $[4,6,9,10,13,14,17,26,31]$ and references therein. Other works where fractures are treated as interfaces include $[5,8,23]$.

The literature on passive transport in fractured porous media and related problems is, however, more scarce. In [28], the authors study a system of advection-diffusion equations where the jump of the diffusive bulk flux acts as a source term inside the fracture. In the coupling conditions, only the diffusive part of the total bulk flux is considered. The discretization is based on the Unfitted Finite Element method, for which well-posedness and $O\left(h^{k}\right)$ convergence in the energy-norm are proved. In [16], a Finite Volume method is combined with a Trace Finite Element method to solve a transport problem in the bulk region and inside the fracture, with the jump of the total bulk flux acting as a source term in the surface problem and under the assumption that the concentration is continuous at the interface. Convergence in $O(h)$ is numerically observed for the energy-norm of the discretization error. A similar problem is studied in [2]. In [27], the authors use an averaging technique similar to [29] in order to derive coupling conditions for a transport problem which allow the concentration to jump across the fracture. This enables them to model high concentration gradients near the fracture resulting from highly heterogeneous diffusivity. The problem is discretized by eXtended Finite Elements (XFEM), and numerical evidence is provided. Another approach can be to consider

\#2, Discrete Fracture Networks (DFNs) models, where the bulk surrounding fractures is considered as comment impervious so that the flow can only occur through the fracture planes and across their intersections; 4 see, e.g., [11], where authors propose a system of unsteady advection-diffusion in DFNs.

In this work, we consider the passive transport of a solute driven by a velocity field solution of a (decoupled) Darcy problem. We present two novel contributions:

(i) first, we propose new coupling conditions between the bulk region and fracture inspired by energy-based arguments, following the general ideas developed by [25] in a different context. Crucially, these transmission conditions allow the solute concentration to jump across the fracture;

(ii) second, we propose a novel HHO discretization of this new model where the Darcy velocity field results from an $\mathrm{HHO}$ approximation of the flow problem in the spirit of [15]. The discretization is designed to incorporate the new transmission conditions and to reproduce at the discrete level the energy argument from which they originate. 
The main source of inspiration for the discretization of the advection terms in the bulk region and inside the fracture is [20], where the authors develop an HHO method that is proven to be

Referee

$\# 3$,

typos

Referee

$\# 3$,

comment

\section{$\begin{array}{lll}4 & 2 & \text { The differential model }\end{array}$}

In this section we introduce the strong and weak formulations of the flow and passive transport problems in the steady case. For the sake of simplicity, the presentation focuses on the two-dimensional case with a single fracture.

\subsection{Notation}

We consider a porous medium saturated by an incompressible fluid that occupies a space region $\Omega \subset \mathbb{R}^{2}$ traversed by a fracture $\Gamma$. We assume that $\Omega$ is an open, bounded, connected, polygonal set with Lipschitz boundary $\partial \Omega$. The fracture $\Gamma$ is represented by an open line segment of nonzero length which cuts $\Omega$ into two disjoint connected polygonal subdomains $\Omega_{\mathrm{B}, 1}$ and $\Omega_{\mathrm{B}, 2}$ with Lipschitz boundary. The set $\Omega_{\mathrm{B}}:=\Omega \backslash \bar{\Gamma}=\Omega_{\mathrm{B}, 1} \cup \Omega_{\mathrm{B}, 2}$ corresponds to the bulk region. We denote by $\partial \Omega_{\mathrm{B}}:=\bigcup_{i=1}^{2}\left(\partial \Omega_{\mathrm{B}, i} \backslash \bar{\Gamma}\right)$ the external boundary of the bulk region and by $\boldsymbol{n}_{\partial \Omega}$ the unit normal vector on $\partial \Omega_{\mathrm{B}}$ pointing out of $\Omega_{\mathrm{B}}$. For $i \in\{1,2\}$, we let $\partial \Omega_{\mathrm{B}, i}:=\partial \Omega_{\mathrm{B}} \cap \overline{\Omega_{\mathrm{B}, i}}$ denote the external boundary of the subdomain $\Omega_{\mathrm{B}, i}$. The boundary of the fracture $\Gamma$ is denoted by $\partial \Gamma$, and the corresponding outward unit tangential vector is $\boldsymbol{\tau}_{\partial \Gamma}$. Finally, $\boldsymbol{n}_{\Gamma}$ denotes the unit normal vector to $\Gamma$ pointing out of $\Omega_{\mathrm{B}, 1}$. This notation is illustrated in Figure 1 .

For any function $\varphi$ sufficiently regular to admit a (possibly two-valued) trace on $\Gamma$, we define the jump and average operators such that

$$
\llbracket \varphi \rrbracket_{\Gamma}:=\left(\varphi_{1}-\varphi_{2}\right)_{\mid \Gamma}, \quad\{\{\varphi\}\}_{\Gamma}:=\left(\frac{\varphi_{1}+\varphi_{2}}{2}\right)_{\mid \Gamma},
$$

where $\varphi_{i}:=\varphi_{\mid \Omega_{\mathrm{B}, i}}$ denotes the restriction of $\varphi$ to the subdomain $\Omega_{\mathrm{B}, i} \subset \Omega_{\mathrm{B}}$. When applied to vector-valued functions, these operators act component-wise.

Finally, for any $X \subset \bar{\Omega}$, we denote by $(\cdot, \cdot)_{X}$ and $\|\cdot\|_{X}$ the usual inner product and norm of $L^{2}(X)$ or $L^{2}(X)^{2}$, according to the context.
Referee

\#3,

Represee

$\# 1$,

comment

1

Referee

\#3,

comment

4

Referee

\# 3 ,

comment

4

Referee

\#1,

comment

2 


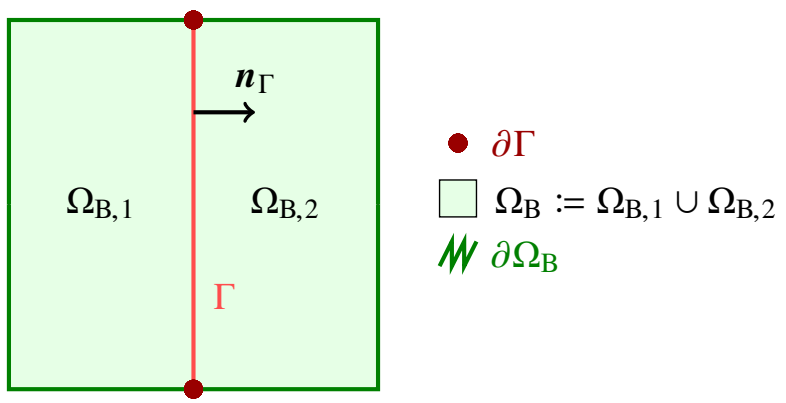

Figure 1: Illustration of the notation introduced in Section 2.1.

\subsection{Darcy flow}

We now formulate the equations that govern the flow in the saturated, fractured porous medium and discuss a weak formulation inspired by $[7,18]$.

\subsubsection{Governing equations}

In the bulk region $\Omega_{\mathrm{B}}$ and in the fracture $\Gamma$, we model the fluid flow by Darcy's law in mixed and primal form, respectively, so that the bulk Darcy velocity $u: \Omega_{\mathrm{B}} \rightarrow \mathbb{R}^{2}$, the bulk pressure $p: \Omega_{\mathrm{B}} \rightarrow \mathbb{R}$, and the fracture pressure $p_{\Gamma}: \Gamma \rightarrow \mathbb{R}$ satisfy

$$
\begin{aligned}
\boldsymbol{u}+\boldsymbol{K} \nabla p & =0 & & \text { in } \Omega_{\mathrm{B}}, \\
\nabla \cdot \boldsymbol{u} & =f & & \text { in } \Omega_{\mathrm{B}}, \\
-\nabla_{\tau} \cdot\left(K_{\Gamma} \nabla_{\tau} p_{\Gamma}\right) & =\ell_{\Gamma} f_{\Gamma}+\llbracket \boldsymbol{u} \rrbracket_{\Gamma} \cdot \boldsymbol{n}_{\Gamma} & & \text { in } \Gamma, \\
\boldsymbol{u} \cdot \boldsymbol{n}_{\partial \Omega} & =0 & & \text { on } \partial \Omega_{\mathrm{B}}, \\
-K_{\Gamma} \nabla_{\tau} p_{\Gamma} \cdot \tau_{\partial \Gamma} & =0 & & \text { on } \partial \Gamma, \\
\int_{\Gamma} p_{\Gamma} & =0, & &
\end{aligned}
$$

where $f \in L^{2}\left(\Omega_{\mathrm{B}}\right)$ and $f_{\Gamma} \in L^{2}(\Gamma)$ denote source or sink terms, $\boldsymbol{K}: \Omega_{\mathrm{B}} \rightarrow \mathbb{R}^{2 \times 2}$ the bulk permeability tensor, and we have set $K_{\Gamma}:=\kappa_{\Gamma}^{\tau} \ell_{\Gamma}$, with $\kappa_{\Gamma}^{\tau}: \Gamma \rightarrow \mathbb{R}$ denoting the tangential permeability inside the fracture and $\ell_{\Gamma}: \Gamma \rightarrow \mathbb{R}$ the fracture thickness. In (1c) and (1e), $\nabla_{\tau}$ and $\nabla_{\tau} \cdot$ denote the tangential gradient and divergence operators along $\Gamma$, respectively. We assume that $\boldsymbol{K}$ is symmetric, piecewise constant on a finite polygonal partition

$$
\mathcal{P}_{\mathrm{B}}=\left\{\omega_{\mathrm{B}, i}: i \in I_{\mathrm{B}}\right\}
$$

of $\Omega_{\mathrm{B}}$, and uniformly elliptic, so that there exist two strictly positive real numbers $\underline{K}_{\mathrm{B}}$ and $\bar{K}_{\mathrm{B}}$ such that, for almost every $\boldsymbol{x} \in \Omega_{\mathrm{B}}$ and all $z \in \mathbb{R}^{2}$ with $|z|=1$,

$$
0<\underline{K}_{\mathrm{B}} \leq \boldsymbol{K}(\boldsymbol{x}) z \cdot z \leq \bar{K}_{\mathrm{B}} .
$$

The quantities $\kappa_{\Gamma}^{\tau}$ and $\ell_{\Gamma}$ are also assumed piecewise constant on a finite partition

$$
\mathcal{P}_{\Gamma}=\left\{\omega_{\Gamma, i}: i \in I_{\Gamma}\right\}
$$

of $\Gamma$, and such that there exist strictly positive real numbers $\underline{\ell}_{\Gamma}, \bar{\ell}_{\Gamma} \underline{K}_{\Gamma}, \bar{K}_{\Gamma}$ such that, for almost every $\boldsymbol{x} \in \Gamma$,

$$
0<\underline{\ell}_{\Gamma} \leq \ell_{\Gamma}(\boldsymbol{x}) \leq \bar{\ell}_{\Gamma}, \quad 0<\underline{K}_{\Gamma} \leq K_{\Gamma}(\boldsymbol{x}) \leq \bar{K}_{\Gamma} .
$$


To close the problem, we add the following transmission conditions across the fracture:

$$
\begin{aligned}
\lambda_{\Gamma}\{\{\boldsymbol{u}\}\}_{\Gamma} \cdot \boldsymbol{n}_{\Gamma} & =\llbracket p \rrbracket_{\Gamma} & & \text { on } \Gamma, \\
\lambda_{\Gamma}^{\xi} \llbracket \boldsymbol{u} \rrbracket_{\Gamma} \cdot \boldsymbol{n}_{\Gamma} & =\{\{p\}\}_{\Gamma}-p_{\Gamma} & & \text { on } \Gamma,
\end{aligned}
$$

where, denoting by $\xi \in\left(\frac{1}{2}, 1\right]$ a user-dependent model parameter, we have set

$$
\lambda_{\Gamma}:=\frac{\ell_{\Gamma}}{\kappa_{\Gamma}^{n}}, \quad \lambda_{\Gamma}^{\xi}:=\lambda_{\Gamma}\left(\frac{\xi}{2}-\frac{1}{4}\right) .
$$

Here, $\kappa_{\Gamma}^{n}: \Gamma \rightarrow \mathbb{R}$ represents the normal permeability inside the fracture, which is assumed piecewise constant on the partition $\mathcal{P}_{\Gamma}$ of $\Gamma$ and such that, for almost every $\boldsymbol{x} \in \Gamma$,

$$
0<\underline{\lambda}_{\Gamma} \leq \lambda_{\Gamma}(\boldsymbol{x}) \leq \bar{\lambda}_{\Gamma}
$$

for two given strictly positive real numbers $\underline{\lambda}_{\Gamma}$ and $\bar{\lambda}_{\Gamma}$.

Remark 1 (Compatibility condition). Since homogeneous Neumann boundary conditions are considered on both the bulk and fracture boundaries (cf. (1d) and (1e)), the flow through the porous medium is entirely driven by the source terms $f$ and $f_{\Gamma}$, which typically model injection or production wells according to their sign. Decomposing $f$ and $f_{\Gamma}$ into their positive and negative parts, i.e., writing $f=f^{+}-f^{-}$and $f_{\Gamma}=f_{\Gamma}^{+}-f_{\Gamma}^{-}$with $f^{ \pm}:=\frac{|f| \pm f}{2}$ and $f_{\Gamma}^{ \pm}:=\frac{\left|f_{\Gamma}\right| \pm f_{\Gamma}}{2}$, we need to further assume the following compatibility condition in order to ensure that a global mass balance is satisfied:

$$
\int_{\Omega_{\mathrm{B}}} f^{+}+\int_{\Gamma} \ell_{\Gamma} f_{\Gamma}^{+}=\int_{\Omega_{\mathrm{B}}} f^{-}+\int_{\Gamma} \ell_{\Gamma} f_{\Gamma}^{-}
$$

which translates the fact that all the fluid that enters the domain through injection wells must exit the domain through production wells. In this configuration, the fracture pressure $p_{\Gamma}$ is defined up to a constant that is fixed by the zero-average constraint (1f). The bulk pressure, on the other hand, is uniquely defined without additional conditions owing to the coupling conditions (4).

Remark 2 (Boundary conditions). More general boundary conditions can be considered. One can consider, e.g., non-homogeneous Neumann boundary conditions on the bulk and, in the case where fractures hit the domain boundary $\partial \Omega$, non-homogeneous Neumann boundary conditions on the fracture tip can be considered as well. On the other hand, if the fracture boundaries lie in the interior of the domain $\Omega$, a no-flow condition is required, where suitable compatibility conditions have to be enforced in each case. We do not dwelve further into this topic here, as the extension of the proposed method is relatively standard.

\subsubsection{Weak formulation}

We define the space $\boldsymbol{H}\left(\operatorname{div} ; \Omega_{\mathrm{B}}\right.$ ), spanned by vector-valued functions on $\Omega_{\mathrm{B}}$ whose restriction to every bulk subregion $\Omega_{\mathrm{B}, i}, i \in\{1,2\}$, is in $\boldsymbol{H}\left(\operatorname{div} ; \Omega_{\mathrm{B}, i}\right)$. The Darcy velocity space is

$$
\boldsymbol{U}:=\left\{\boldsymbol{u} \in \boldsymbol{H}\left(\operatorname{div} ; \Omega_{\mathrm{B}}\right): \boldsymbol{u} \cdot \boldsymbol{n}_{\partial \Omega}=0 \text { on } \partial \Omega_{\mathrm{B}} \text { and }\left(\boldsymbol{u}_{1} \cdot \boldsymbol{n}_{\Gamma}, \boldsymbol{u}_{2} \cdot \boldsymbol{n}_{\Gamma}\right) \in L^{2}(\Gamma)^{2}\right\} .
$$

The fracture pressure space is $P_{\Gamma}:=H^{1}(\Gamma) \cap L_{0}^{2}(\Gamma)$, with $L_{0}^{2}(\Gamma)$ spanned by square-integrable functions with zero mean value on $\Gamma$. We define the bilinear forms $a_{K}^{\xi}: \boldsymbol{U} \times \boldsymbol{U} \rightarrow \mathbb{R}, a_{K}^{\Gamma}: H^{1}(\Gamma) \times H^{1}(\Gamma) \rightarrow \mathbb{R}$, $b: \boldsymbol{U} \times L^{2}\left(\Omega_{\mathrm{B}}\right) \rightarrow \mathbb{R}$ and $d: \boldsymbol{U} \times L^{2}(\Gamma) \rightarrow \mathbb{R}$ such that

$$
\begin{gathered}
a_{\boldsymbol{K}}^{\xi}(\boldsymbol{u}, \boldsymbol{q}):=\left(\boldsymbol{K}^{-1} \boldsymbol{u}, \boldsymbol{q}\right)_{\Omega_{\mathrm{B}}}+\left(\lambda_{\Gamma}^{\xi} \llbracket \boldsymbol{u} \rrbracket_{\Gamma} \cdot \boldsymbol{n}_{\Gamma}, \llbracket \boldsymbol{q} \rrbracket_{\Gamma} \cdot \boldsymbol{n}_{\Gamma}\right)_{\Gamma}+\left(\lambda_{\Gamma}\{\{\boldsymbol{u}\}\}_{\Gamma} \cdot \boldsymbol{n}_{\Gamma},\{\{\boldsymbol{q}\}\}_{\Gamma} \cdot \boldsymbol{n}_{\Gamma}\right)_{\Gamma}, \\
a_{K}^{\Gamma}\left(p_{\Gamma}, z_{\Gamma}\right):=\left(K_{\Gamma} \nabla_{\tau} p_{\Gamma}, \nabla_{\tau} z_{\Gamma}\right)_{\Gamma}, \quad b(\boldsymbol{u}, z):=(\nabla \cdot \boldsymbol{u}, z)_{\Omega_{\mathrm{B}}}, \quad d\left(\boldsymbol{u}, z_{\Gamma}\right):=\left(\llbracket \boldsymbol{u} \rrbracket_{\Gamma} \cdot \boldsymbol{n}_{\Gamma}, z_{\Gamma}\right)_{\Gamma},
\end{gathered}
$$


as well as the global bilinear form $\mathcal{A}_{\xi}^{\text {flow }}:\left(\boldsymbol{U} \times L^{2}\left(\Omega_{\mathrm{B}}\right) \times H^{1}(\Gamma)\right) \times\left(\boldsymbol{U} \times L^{2}\left(\Omega_{\mathrm{B}}\right) \times H^{1}(\Gamma)\right) \rightarrow \mathbb{R}$ such that

$$
\mathcal{A}_{\xi}^{\text {flow }}\left(\left(\boldsymbol{u}, p, p_{\Gamma}\right),\left(\boldsymbol{q}, z, z_{\Gamma}\right)\right):=a_{K}^{\xi}(\boldsymbol{u}, \boldsymbol{q})+b(\boldsymbol{u}, z)-b(\boldsymbol{q}, p)+d\left(\boldsymbol{q}, p_{\Gamma}\right)-d\left(\boldsymbol{u}, z_{\Gamma}\right)+a_{K}^{\Gamma}\left(p_{\Gamma}, z_{\Gamma}\right) .
$$

With these spaces and bilinear forms, the weak formulation of problem (1)-(4) reads: Find $\left(\boldsymbol{u}, p, p_{\Gamma}\right) \in$ $\boldsymbol{U} \times L^{2}\left(\Omega_{\mathrm{B}}\right) \times P_{\Gamma}$ such that, for all $\left(\boldsymbol{q}, z, z_{\Gamma}\right) \in \boldsymbol{U} \times L^{2}\left(\Omega_{\mathrm{B}}\right) \times H^{1}(\Gamma)$,

$$
\mathcal{A}_{\xi}^{\text {flow }}\left(\left(\boldsymbol{u}, p, p_{\Gamma}\right),\left(\boldsymbol{q}, z, z_{\Gamma}\right)\right)=(f, z)_{\Omega_{\mathrm{B}}}+\left(\ell_{\Gamma} f_{\Gamma}, z_{\Gamma}\right)_{\Gamma} .
$$

The well-posedness of problem (6) with mixed boundary conditions is studied in [7]; cf. also [18, 31] and references therein.

\subsection{Passive transport}

We next formulate the equations that govern the passive transport of a solute by the Darcy flow solution of problem (1)-(4). For the sake of simplicity, we focus on the case where the transport problem is fully decoupled. This section contains the first main contribution of this paper, namely novel transmission conditions that enable the treatment of discontinuous solute concentrations across the fracture.

\subsubsection{Bulk region}

Denoting by $c: \Omega_{\mathrm{B}} \rightarrow \mathbb{R}$ the concentration of the solute in the bulk and by $\boldsymbol{D}: \Omega_{\mathrm{B}} \rightarrow \mathbb{R}^{2 \times 2}$ the symmetric, uniformly elliptic bulk diffusion-dispersion tensor, the passive transport of the solute in the bulk region is governed by the following equations:

$$
\begin{aligned}
\nabla \cdot(\boldsymbol{u} c-\boldsymbol{D} \nabla c)+f^{-} c & =f^{+} \widehat{c} & & \text { in } \Omega_{\mathrm{B}}, \\
-\boldsymbol{D} \nabla c \cdot \boldsymbol{n}_{\partial \Omega} & =0 & & \text { on } \partial \Omega_{\mathrm{B}},
\end{aligned}
$$

where the term $f^{-} c$ acts as a sink, while the term $f^{+} \widehat{c}$, with $\widehat{c}: \Omega_{\mathrm{B}} \rightarrow \mathbb{R}$ denoting the concentration of solute as it is injected, acts as a source. We assume that both $\boldsymbol{D}$ and $\widehat{c}$ are piecewise constant on the polygonal partition $\mathcal{P}_{\mathrm{B}}$ of $\Omega_{\mathrm{B}}$ (see (2)), and that there exist two strictly positive real numbers $\underline{D}_{\mathrm{B}}$ and $\bar{D}_{\mathrm{B}}$ such that, for almost every $\boldsymbol{x} \in \Omega_{\mathrm{B}}$ and all $z \in \mathbb{R}^{2}$ such that $|z|=1$,

$$
0 \leq \widehat{c}(\boldsymbol{x}) \leq 1, \quad 0<\underline{D}_{\mathrm{B}} \leq \boldsymbol{D}(\boldsymbol{x}) \boldsymbol{z} \cdot \boldsymbol{z} \leq \bar{D}_{\mathrm{B}} .
$$

More generally $\boldsymbol{D}$ can depend on $\boldsymbol{u}$. While the theoretical results provided hereafter focus on the case of $\boldsymbol{D}$ independent from $\boldsymbol{u}$, this dependence has been considered in some numerical experiments presented in Section 5.

\subsubsection{Fracture}

We define the Darcy velocity $\boldsymbol{u}_{\Gamma}: \Gamma \rightarrow \mathbb{R}^{2}$ inside the fracture such that $\boldsymbol{u}_{\Gamma}:=-K_{\Gamma} \nabla_{\tau} p_{\Gamma}$ where $p_{\Gamma}: \Gamma \rightarrow \mathbb{R}$ is the fracture pressure solution of problem (1)-(4). Denoting by $c_{\Gamma}: \Gamma \rightarrow \mathbb{R}$ the concentration of the solute inside the fracture, and letting $D_{\Gamma}:=\mathcal{D}_{\Gamma}^{\tau} \ell_{\Gamma}$ with $\mathcal{D}_{\Gamma}^{\tau}: \Gamma \rightarrow \mathbb{R}$ denoting the (strictly positive almost everywhere) tangential diffusion-dispersion coefficient of the fracture, the governing equations for the transport problem inside the fracture are:

$$
\begin{aligned}
\nabla_{\boldsymbol{\tau}} \cdot\left(\boldsymbol{u}_{\Gamma} c_{\Gamma}-D_{\Gamma} \nabla_{\tau} c_{\Gamma}\right)+\ell_{\Gamma} f_{\Gamma}^{-} c_{\Gamma} & =\ell_{\Gamma} f_{\Gamma}^{+} \widehat{c_{\Gamma}}+\llbracket \boldsymbol{u} c-\boldsymbol{D} \nabla c \rrbracket_{\Gamma} \cdot \boldsymbol{n}_{\Gamma} & & \text { in } \Gamma, \\
-D_{\Gamma} \nabla_{\tau} c_{\Gamma} \cdot \boldsymbol{\tau}_{\partial \Gamma} & =0 & & \text { on } \partial \Gamma,
\end{aligned}
$$

Referee

Referee

$\# 1$,

comment 
where again $f_{\Gamma}^{-} c$ acts as a sink term while $f_{\Gamma}^{+} \widehat{c_{\Gamma}}$ acts as a source, with $\widehat{c_{\Gamma}}: \Gamma \rightarrow \mathbb{R}$ denoting the concentration of solute as it is injected into the fracture. For the sake of simplicity, we assume in what follows that both $\widehat{c_{\Gamma}}$ and $D_{\Gamma}$ are piecewise constant on the partition $\mathcal{P}_{\Gamma}$ of $\Gamma$ (see (3)), and such that there exist two strictly positive real numbers $\underline{D}_{\Gamma}$ and $\bar{D}_{\Gamma}$ such that, for almost every $\boldsymbol{x} \in \Gamma$,

$$
0 \leq \widehat{c_{\Gamma}}(\boldsymbol{x}) \leq 1, \quad 0<\underline{D}_{\Gamma} \leq D_{\Gamma}(\boldsymbol{x}) \leq \bar{D}_{\Gamma} .
$$

Remark 3 (Bulk and fracture boundary conditions). Considering no-flow boundary conditions on the bulk and fracture flux (1d) and (1e) entails a slight simplification, since we do not have to deal with the decomposition of the bulk or fracture boundary into their respective inflow or outflow parts.

Referee \#2, comment 1

\subsubsection{Transmission conditions}

To derive transmission conditions for the hybrid dimensional passive transport problem, we have followed a technique similar to that used in [25] in a completely different context. We started from the observation that in the unreduced model, where the fracture is not reduced to an internal interface, the transport operator does not contribute to the energy balance, a part possible contributions at the boundary (which are zero in the case of zero normal velocity at the boundary). Therefore, we want to obtain also in the reduced model an energy estimate where the transport term behaves similarly. In particular, in the energy estimate for the hybrid dimensional problem the terms related to transport in the coupling conditions have to cancel out and give no contribution to the energy. This is crucial since energy estimates are a key ingredient for the coercivity of the differential problem. This is far from trivial, however we will show that this is possible if the following conditions are used (see Theorem 6):

$$
\begin{array}{cr}
\{\{\boldsymbol{u} c-\boldsymbol{D} \nabla c\}\}_{\Gamma} \cdot \boldsymbol{n}_{\Gamma}=\beta_{\Gamma} \llbracket c \rrbracket_{\Gamma}+\left(\{\{\boldsymbol{u}\}\}_{\Gamma} \cdot \boldsymbol{n}_{\Gamma}\right)\{\{c\}\}_{\Gamma}+\frac{1}{8}\left(\llbracket \boldsymbol{u} \rrbracket_{\Gamma} \cdot \boldsymbol{n}_{\Gamma}\right) \llbracket c \rrbracket_{\Gamma} & \text { on } \Gamma, \\
\llbracket \boldsymbol{u} c-\boldsymbol{D} \nabla c \rrbracket_{\Gamma} \cdot \boldsymbol{n}_{\Gamma}=\beta_{\Gamma}^{\xi}\left(\{\{c\}\}_{\Gamma}-c_{\Gamma}\right)+\frac{1}{2}\left(\llbracket \boldsymbol{u} \rrbracket_{\Gamma} \cdot \boldsymbol{n}_{\Gamma}\right)\left(\{\{c\}\}_{\Gamma}+c_{\Gamma}\right) & \text { on } \Gamma,
\end{array}
$$

where $\xi$ is the user-dependent model parameter introduced in Section 2.2.1, and we have set

$$
\beta_{\Gamma}:=\frac{\mathcal{D}_{\Gamma}^{n}}{\ell_{\Gamma}}, \quad \beta_{\Gamma}^{\xi}:=\beta_{\Gamma}\left(\frac{\xi}{2}-\frac{1}{4}\right)^{-1} .
$$

The term $\mathcal{D}_{\Gamma}^{n}: \Gamma \rightarrow \mathbb{R}$ represents the normal diffusion-dispersion coefficient of the fracture, which is assumed piecewise constant on the partition $\mathcal{P}_{\Gamma}$ of $\Gamma$ (see (3)), strictly positive almost everywhere on $\Gamma$, and such that, for almost every $\boldsymbol{x} \in \Gamma$,

$$
0<\underline{\beta}_{\Gamma} \leq \beta_{\Gamma}(\boldsymbol{x}) \leq \bar{\beta}_{\Gamma},
$$

Referee \#3, comment 6

Referee \#1, comment 5

Referee \#1, comment

$$
\begin{aligned}
\{\{-\boldsymbol{D} \nabla c\}\}_{\Gamma} \cdot \boldsymbol{n}_{\Gamma} & =\beta_{\Gamma} \llbracket c \rrbracket_{\Gamma} & & \text { on } \Gamma, \\
\llbracket-\boldsymbol{D} \nabla c \rrbracket_{\Gamma} \cdot \boldsymbol{n}_{\Gamma} & =\beta_{\Gamma}^{\xi}\left(\{\{c\}\}_{\Gamma}-c_{\Gamma}\right) & & \text { on } \Gamma .
\end{aligned}
$$

The first equation (10a) stipulates that the diffusive flux across the fracture is proportional to the difference of concentration at the two sides of the fracture, while the second equation (10b) stipulates 
that the exchange between the bulk and the fracture is proportional to the difference between the average concentration across the fracture and the concentation at the interior of the fracture. For a pure transport problem, which corresponds to the case when all the diffusion-dispersion coefficients are zero, simple algebraic manipulations show that we obtain $c_{1}=c_{2}=c_{\Gamma}$ across the fracture $\Gamma$, provided that $\llbracket u \rrbracket_{\Gamma} \cdot \boldsymbol{n}_{\Gamma} \neq 0$. This is reasonable, since in this limit case concentration is just transmitted across the interface. In the intermediate case, the transmission conditions (9) as designed so as to guarantee the satisfaction of the energy inequality, as we will show in Section 2.3.5.

\subsubsection{Weak formulation}

Let $H^{1}\left(\Omega_{\mathrm{B}}\right)$ denote the broken Sobolev space spanned by scalar-valued functions on $\Omega_{\mathrm{B}}$ whose restriction to every bulk subregion $\Omega_{\mathrm{B}, i}, i \in\{1,2\}$, is in $H^{1}\left(\Omega_{\mathrm{B}, i}\right)$. We define the molecular diffusion bilinear form $a_{D}: H^{1}\left(\Omega_{\mathrm{B}}\right) \times H^{1}\left(\Omega_{\mathrm{B}}\right) \rightarrow \mathbb{R}$, the advection-reaction bilinear form $a_{\boldsymbol{u}, f}: H^{1}\left(\Omega_{\mathrm{B}}\right) \times$ $H^{1}\left(\Omega_{\mathrm{B}}\right) \rightarrow \mathbb{R}$, and the diffusion-advection-reaction bilinear form $a: H^{1}\left(\Omega_{\mathrm{B}}\right) \times H^{1}\left(\Omega_{\mathrm{B}}\right)$ such that

$$
\begin{gathered}
a_{D}(c, z):=\int_{\Omega_{\mathrm{B}}} D \nabla c \cdot \nabla z, \quad a_{\boldsymbol{u}, f}(c, z):=\int_{\Omega_{\mathrm{B}}}\left(-c(\boldsymbol{u} \cdot \nabla z)+f^{-} c z\right), \\
a(c, z):=a_{D}(c, z)+a_{\boldsymbol{u}, f}(c, z) .
\end{gathered}
$$

We also define their fracture-based counterparts $a_{D}^{\Gamma}: H^{1}(\Gamma) \times H^{1}(\Gamma) \rightarrow \mathbb{R}, a_{\boldsymbol{u}, f}^{\Gamma}: H^{1}(\Gamma) \times H^{1}(\Gamma) \rightarrow \mathbb{R}$ and $a_{\Gamma}: H^{1}(\Gamma) \times H^{1}(\Gamma) \rightarrow \mathbb{R}$ such that

$$
\begin{gathered}
a_{D}^{\Gamma}\left(c_{\Gamma}, z_{\Gamma}\right):=\int_{\Gamma} D_{\Gamma} \nabla_{\tau} c_{\Gamma} \cdot \nabla_{\tau} z_{\Gamma}, \quad a_{\boldsymbol{u}, f}^{\Gamma}\left(c_{\Gamma}, z_{\Gamma}\right):=\int_{\Gamma}\left(-c_{\Gamma}\left(\boldsymbol{u}_{\Gamma} \cdot \nabla_{\tau} z_{\Gamma}\right)+\ell_{\Gamma} f_{\Gamma}^{-} c_{\Gamma} z_{\Gamma}\right), \\
a_{\Gamma}\left(c_{\Gamma}, z_{\Gamma}\right):=a_{D}^{\Gamma}\left(c_{\Gamma}, z_{\Gamma}\right)+a_{\boldsymbol{u}, f}^{\Gamma}\left(c_{\Gamma}, z_{\Gamma}\right) .
\end{gathered}
$$

The global bilinear form $\mathcal{A}_{\xi}^{\text {transp }}:\left(H^{1}\left(\Omega_{\mathrm{B}}\right) \times H^{1}(\Gamma)\right) \times\left(H^{1}\left(\Omega_{\mathrm{B}}\right) \times H^{1}(\Gamma)\right) \rightarrow \mathbb{R}$, that additionally takes into account terms that stem from the coupling equations, is defined as follows:

$$
\begin{aligned}
\mathcal{A}_{\xi}^{\text {transp }}\left(\left(c, c_{\Gamma}\right),\left(z, z_{\Gamma}\right)\right):= & a(c, z)+a_{\Gamma}\left(c_{\Gamma}, z_{\Gamma}\right)+\int_{\Gamma} \beta_{\Gamma}^{\xi}\left(\{\{c\}\}_{\Gamma}-c_{\Gamma}\right)\left(\{\{z\}\}_{\Gamma}-z_{\Gamma}\right) \\
& +\int_{\Gamma}\left(\beta_{\Gamma} \llbracket c \rrbracket_{\Gamma} \llbracket z \rrbracket_{\Gamma}+\frac{1}{2}\left(\llbracket \boldsymbol{u} \rrbracket_{\Gamma} \cdot \boldsymbol{n}_{\Gamma}\right)\left(\{\{c\}\}_{\Gamma}+c_{\Gamma}\right)\left(\{\{z\}\}_{\Gamma}-z_{\Gamma}\right)\right) \\
& +\int_{\Gamma}\left(\left(\{\{\boldsymbol{u}\}\}_{\Gamma} \cdot \boldsymbol{n}_{\Gamma}\right)\{\{c\}\}_{\Gamma} \llbracket z \rrbracket_{\Gamma}+\frac{1}{8}\left(\llbracket \boldsymbol{u} \rrbracket_{\Gamma} \cdot \boldsymbol{n}_{\Gamma}\right) \llbracket c \rrbracket_{\Gamma} \llbracket z \rrbracket_{\Gamma}\right) .
\end{aligned}
$$

With these spaces and bilinear forms, the weak formulation of problem (7)-(8)-(9) reads: Find $\left(c, c_{\Gamma}\right) \in H^{1}\left(\Omega_{\mathrm{B}}\right) \times H^{1}(\Gamma)$ such that, for all $\left(z, z_{\Gamma}\right) \in H^{1}\left(\Omega_{\mathrm{B}}\right) \times H^{1}(\Gamma)$

$$
\mathcal{A}_{\xi}^{\text {transp }}\left(\left(c, c_{\Gamma}\right),\left(z, z_{\Gamma}\right)\right)=\left(f^{+} \widehat{c}, z\right)_{\Omega_{\mathrm{B}}}+\left(\ell_{\Gamma} f_{\Gamma}^{+} \widehat{c_{\Gamma}}, z_{\Gamma}\right)_{\Gamma} .
$$

[DDP: Florent, I think that what the referee wants us to comment on the conservativity of the HHO method, not the continuous problem]

Remark 5 (Local mass conservation). Let a control volume $A \subset \Omega$ such that $A_{\mathrm{B}}:=A \backslash \Gamma \neq \emptyset$ and $A_{\Gamma}:=A \cap \Gamma \neq \emptyset$. Integrating and summing the steady passive transport equations (7a) and (8a) on, respectively, $A_{\mathrm{B}}$ and $A_{\Gamma}$, then using the Green's formula on the divergence and the tangential divergence for, respectively, the bulk and fracture term, it holds

$$
\begin{aligned}
\int_{\partial A_{\mathrm{B}} \backslash \Gamma}(\boldsymbol{u} c-\boldsymbol{D} \nabla \boldsymbol{c}) \cdot \boldsymbol{n}_{\partial A_{\mathrm{B}}} & +\sum_{V \in \partial A_{\Gamma}}\left(\boldsymbol{u}_{\Gamma} c_{\Gamma}-D_{\Gamma} \nabla_{\tau} c_{\Gamma}\right) \cdot \boldsymbol{\tau}_{\partial A_{\Gamma}}(V) \\
& +\int_{A_{\mathrm{B}}} f^{-} c+\int_{A_{\Gamma}} \ell_{\Gamma} f_{\Gamma}^{-} c_{\Gamma}=\int_{A_{\mathrm{B}}} f^{+} \widehat{c}+\int_{A_{\Gamma}} \ell_{\Gamma} f_{\Gamma}^{+} \widehat{c_{\Gamma}},
\end{aligned}
$$


which expresses the fact that the variation of $c$ and $c_{\Gamma}$ in the controle volume $A$ due to the advective and diffusive exchanges through $\partial A$ plus the quantity of $c$ and $c_{\Gamma}$ depleted by reaction over $A$ is equal to the integral of the quantity of $c$ and $c_{\Gamma}$ generated by source terms over $A$.

\subsubsection{Coercivity}

In the following theorem, we prove the coercivity of the global transport bilinear form defined by (13) and show that, thanks to the new transmission conditions (8), the advective terms do not dissipate energy. This result is the key ingredient to derive a stability result for problem (14).

Theorem 6 (Coercivity). Let $\xi>1 / 2$. Then, for all $\left(z, z_{\Gamma}\right) \in H^{1}\left(\Omega_{\mathrm{B}}\right) \times H^{1}(\Gamma)$, it holds

$$
\begin{aligned}
\mathcal{A}_{\xi}^{\text {transp }}\left(\left(z, z_{\Gamma}\right),\left(z, z_{\Gamma}\right)\right)= & \left\|\boldsymbol{D}^{1 / 2} \nabla z\right\|_{\Omega_{\mathrm{B}}}^{2}+\left\|D_{\Gamma}^{1 / 2} \nabla_{\tau} z_{\Gamma}\right\|_{\Gamma}^{2}+\left\|\chi_{\mathrm{B}}^{1 / 2} z\right\|_{\Omega_{\mathrm{B}}}^{2}+\left\|\chi_{\Gamma}^{1 / 2} z_{\Gamma}\right\|_{\Gamma}^{2} \\
& +\left\|\left(\beta_{\Gamma}^{\xi}\right)^{1 / 2}\left(\{\{z\}\}_{\Gamma}-z_{\Gamma}\right)\right\|_{\Gamma}^{2}+\left\|\left(\beta_{\Gamma}\right)^{1 / 2} \llbracket z\right\|_{\Gamma} \|_{\Gamma}^{2},
\end{aligned}
$$

with $\chi_{\mathrm{B}}:=\frac{|f|}{2}$ and $\chi_{\Gamma}:=\frac{\ell_{\Gamma}\left|f_{\Gamma}\right|}{2}$.

Remark 7 (Energy balance). Equation (15) can be interpreted as a global energy balance. The transmission conditions (9) are designed so that the advective terms do not contribute to this balance.

Referee $\# 1, \# 3$, typos,

Additionally, if $z=z_{\Gamma}$ across $\Gamma$, also all terms related to the diffusion-dispersion across the fracture, collected in the second line of (15), disappear.

Proof. Let $\left(z, z_{\Gamma}\right) \in H^{1}\left(\Omega_{\mathrm{B}}\right) \times H^{1}(\Gamma)$. By definition of the global bilinear form $\mathcal{A}_{\xi}^{\text {transp }}$ (13), it holds

Referee

\#2,

typos

$$
\begin{aligned}
\mathcal{A}_{\xi}^{\text {transp }}\left(\left(z, z_{\Gamma}\right),\left(z, z_{\Gamma}\right)\right)= & a(z, z)+a_{\Gamma}\left(z_{\Gamma}, z_{\Gamma}\right)+\left\|\left(\beta_{\Gamma}^{\xi}\right)^{1 / 2}\left(\{\{z\}\}_{\Gamma}-z_{\Gamma}\right)\right\|_{\Gamma}^{2}+\left\|\left(\beta_{\Gamma}\right)^{1 / 2} \llbracket z \rrbracket_{\Gamma}\right\|_{\Gamma}^{2} \\
& +\int_{\Gamma} \frac{1}{2}\left(\llbracket \boldsymbol{u} \rrbracket_{\Gamma} \cdot \boldsymbol{n}_{\Gamma}\right)\left(\{\{z\}\}_{\Gamma}+z_{\Gamma}\right)\left(\{\{z\}\}_{\Gamma}-z_{\Gamma}\right) \\
& +\int_{\Gamma}\left(\left(\{\{\boldsymbol{u}\}\}_{\Gamma} \cdot \boldsymbol{n}_{\Gamma}\right)\{\{z\}\}_{\Gamma} \llbracket z z \|_{\Gamma}+\frac{1}{8}\left(\llbracket \boldsymbol{u} \rrbracket_{\Gamma} \cdot \boldsymbol{n}_{\Gamma}\right) \llbracket z \rrbracket_{\Gamma}^{2}\right),
\end{aligned}
$$

Using the definitions (11) and (12) of the bilinear forms $a$ and $a_{\Gamma}$, we obtain

$$
a(z, z)=\left\|\boldsymbol{D}^{1 / 2} \nabla z\right\|_{\Omega_{\mathrm{B}}}^{2}+a_{\boldsymbol{u}, f}(z, z), \quad a_{\Gamma}\left(z_{\Gamma}, z_{\Gamma}\right)=\left\|D_{\Gamma}^{1 / 2} \nabla_{\tau} z_{\Gamma}\right\|_{\Gamma}^{2}+a_{\boldsymbol{u}, f}^{\Gamma}\left(z_{\Gamma}, z_{\Gamma}\right) .
$$

Expanding the bilinear form $a_{\boldsymbol{u}, f}$ according to its definition (11), we get

$$
\begin{aligned}
a_{\boldsymbol{u}, f}(z, z) & =\int_{\Omega_{\mathrm{B}}}\left(-z(\boldsymbol{u} \cdot \nabla z)+f^{-} z^{2}\right) \\
& =\int_{\Omega_{\mathrm{B}}}\left(-\boldsymbol{u} \cdot \nabla\left(\frac{z^{2}}{2}\right)+f^{-} z^{2}\right) \\
& =\int_{\Omega_{\mathrm{B}}}\left(\frac{1}{2}(\nabla \cdot \boldsymbol{u}) z^{2}+f^{-} z^{2}\right)-\frac{1}{2} \int_{\Gamma} \llbracket \boldsymbol{u} z^{2} \rrbracket_{\Gamma} \cdot \boldsymbol{n}_{\Gamma} \\
& =\left\|\chi_{\mathrm{B}}^{1 / 2} z\right\|_{\Omega_{\mathrm{B}}}^{2}-\frac{1}{2} \int_{\Gamma}\left(\llbracket \boldsymbol{u} \rrbracket_{\Gamma} \cdot \boldsymbol{n}_{\Gamma}\left\{\left\{z^{2}\right\}\right\}_{\Gamma}+\{\{\boldsymbol{u}\}\}_{\Gamma} \cdot \boldsymbol{n}_{\Gamma} \llbracket z^{2} \rrbracket_{\Gamma}\right),
\end{aligned}
$$

where we have used an integration by parts together with the boundary condition (1d) to pass to the third line while to pass to the fourth line, we have used (1b) to write $\frac{1}{2}(\nabla \cdot \boldsymbol{u})+f^{-}=\frac{f}{2}+f^{-}=\frac{|f|}{2}$ followed by the relation

$$
\llbracket a b \rrbracket_{\Gamma}=\llbracket a \rrbracket_{\Gamma}\{\{b\}\}_{\Gamma}+\{\{a\}\}_{\Gamma} \llbracket b \rrbracket_{\Gamma} .
$$


Similarly, expanding $a_{\boldsymbol{u}, f}^{\Gamma}$ according to its definition (12), we find

$$
\begin{aligned}
a_{\boldsymbol{u}, f}^{\Gamma}\left(z_{\Gamma}, z_{\Gamma}\right) & =\int_{\Gamma}\left(-z_{\Gamma}\left(\boldsymbol{u}_{\Gamma} \cdot \nabla_{\tau} z_{\Gamma}\right)+\ell_{\Gamma} f_{\Gamma}^{-} z_{\Gamma}^{2}\right) \\
& =\int_{\Gamma}\left(-\boldsymbol{u}_{\Gamma} \cdot \nabla\left(\frac{z_{\Gamma}^{2}}{2}\right)+\ell_{\Gamma} f_{\Gamma}^{-} z_{\Gamma}^{2}\right) \\
& =\int_{\Gamma}\left(\frac{1}{2}\left(\nabla_{\tau} \cdot \boldsymbol{u}_{\Gamma}\right) z_{\Gamma}^{2}+\ell_{\Gamma} f_{\Gamma}^{-} z_{\Gamma}^{2}\right) \\
& =\int_{\Gamma}\left(\frac{1}{2}\left(\ell_{\Gamma} f_{\Gamma}+\llbracket \boldsymbol{u} \rrbracket_{\Gamma} \cdot \boldsymbol{n}_{\Gamma}\right) z_{\Gamma}^{2}+\ell_{\Gamma} f_{\Gamma}^{-} z_{\Gamma}^{2}\right) \\
& =\left\|\chi_{\Gamma}^{1 / 2} z_{\Gamma}\right\|_{\Gamma}^{2}+\frac{1}{2} \int_{\Gamma}\left(\llbracket \boldsymbol{u} \rrbracket_{\Gamma} \cdot \boldsymbol{n}_{\Gamma}\right) z_{\Gamma}^{2},
\end{aligned}
$$

where we have, at first, integrated by parts and used (1e) to pass to the third line, then we have used (1c) after recalling that $\boldsymbol{u}_{\Gamma}:=-K_{\Gamma} \nabla_{\tau} p_{\Gamma}$ to pass to the fourth line, and invoked the definition of $\chi_{\Gamma}$ to conclude. Plugging (17), (18) and (20) into (16), we obtain

Referee \#3, typos

$$
\begin{aligned}
\mathcal{A}_{\xi}^{\text {transp }}\left(\left(z, z_{\Gamma}\right),\left(z, z_{\Gamma}\right)\right)= & \left\|\boldsymbol{D}^{1 / 2} \nabla z\right\|_{\Omega_{\mathrm{B}}}^{2}+\left\|D_{\Gamma}^{1 / 2} \nabla_{\tau} z_{\Gamma}\right\|_{\Gamma}^{2}+\left\|\chi_{\mathrm{B}}^{1 / 2} z\right\|_{\Omega_{\mathrm{B}}}^{2}+\left\|\chi_{\Gamma}^{1 / 2} z_{\Gamma}\right\|_{\Gamma}^{2} \\
& +\left\|\left(\beta_{\Gamma}^{\xi}\right)^{1 / 2}\left(\{\{z\}\}_{\Gamma}-z_{\Gamma}\right)\right\|_{\Gamma}^{2}+\left\|\left(\beta_{\Gamma}\right)^{1 / 2} \llbracket z \rrbracket_{\Gamma}\right\|_{\Gamma}^{2} \\
& +\int_{\Gamma}\left(-\frac{1}{2}\left(\llbracket \boldsymbol{u} \rrbracket_{\Gamma} \cdot \boldsymbol{n}_{\Gamma}\right)\left\{\left\{z^{2}\right\}\right\}_{\Gamma}+\left(\{\{\boldsymbol{u}\}\}_{\Gamma} \cdot \boldsymbol{n}_{\Gamma}\right)\left(\left\{\{z\}_{\Gamma} \llbracket z \rrbracket_{\Gamma}-\frac{1}{2} \llbracket z^{2} \rrbracket_{\Gamma}\right)\right)\right. \\
& +\int_{\Gamma} \frac{1}{2}\left(\left(\llbracket \boldsymbol{u} \rrbracket_{\Gamma} \cdot \boldsymbol{n}_{\Gamma}\right) z_{\Gamma}^{2}+\left(\llbracket \boldsymbol{u} \rrbracket_{\Gamma} \cdot \boldsymbol{n}_{\Gamma}\right)\left(\{\{z\}\}_{\Gamma}+z_{\Gamma}\right)\left(\left\{\{z\}_{\Gamma}-z_{\Gamma}\right)\right)\right. \\
& +\int_{\Gamma} \frac{1}{8}\left(\llbracket \boldsymbol{u} \rrbracket_{\Gamma} \cdot \boldsymbol{n}_{\Gamma}\right) \llbracket z \rrbracket_{\Gamma}^{2},
\end{aligned}
$$

where, to cancel the last term in the third line, we have used formula (19) with $a=b=z$ to infer $\frac{1}{2} \llbracket z^{2} \rrbracket_{\Gamma}=\{\{z\}\}_{\Gamma} \llbracket z \rrbracket_{\Gamma}$. Rearranging the terms on $\Gamma$, we arrive at

$$
\begin{aligned}
\mathcal{A}_{\xi}^{\text {transp }}\left(\left(z, z_{\Gamma}\right),\left(z, z_{\Gamma}\right)\right)= & \left\|\boldsymbol{D}^{1 / 2} \nabla z\right\|_{\Omega_{\mathrm{B}}}^{2}+\left\|D_{\Gamma}^{1 / 2} \nabla_{\tau} z_{\Gamma}\right\|_{\Gamma}^{2}+\left\|\chi_{\mathrm{B}}^{1 / 2} z\right\|_{\Omega_{\mathrm{B}}}^{2}+\left\|\chi_{\Gamma}^{1 / 2} z_{\Gamma}\right\|_{\Gamma}^{2} \\
& +\left\|\left(\beta_{\Gamma}^{\xi}\right)^{1 / 2}\left(\{\{z\}\}_{\Gamma}-z_{\Gamma}\right)\right\|_{\Gamma}^{2}+\left\|\left(\beta_{\Gamma}\right)^{1 / 2} \llbracket z\right\|_{\Gamma} \|_{\Gamma}^{2} \\
& +\int_{\Gamma} \frac{1}{2}\left(\llbracket \boldsymbol{u} \rrbracket_{\Gamma} \cdot \boldsymbol{n}_{\Gamma}\right)\left(z_{\Gamma}^{z}-\left\{\left\{z^{2}\right\}\right\}_{\Gamma}+\{\{z\}\}_{\Gamma}^{2}-z_{\Gamma}^{\chi}+\frac{1}{4} \llbracket z \|_{\Gamma}^{2}\right) .
\end{aligned}
$$

Using the formula

$$
\{\{a b\}\}_{\Gamma}=\{\{a\}\}_{\Gamma}\{\{b\}\}_{\Gamma}+\frac{1}{4} \llbracket a \rrbracket_{\Gamma} \llbracket b \rrbracket_{\Gamma}
$$

with $a=b=z$ to write $\left\{\left\{z^{2}\right\}\right\}_{\Gamma}=\{\{z\}\}_{\Gamma}^{2}+\frac{1}{4} \llbracket z \rrbracket_{\Gamma}^{2}$ in the last line of (21), (15) follows.

\section{Discrete setting}

The HHO method is built upon a polygonal mesh of the domain $\Omega$ defined prescribing a set of mesh elements $\mathcal{T}_{h}$ and a set of mesh faces $\mathcal{F}_{h}$.

The set of mesh elements $\mathcal{T}_{h}$ is a finite collection of open disjoint polygons with nonzero area such that $\bar{\Omega}=\bigcup_{T \in \mathcal{T}_{h}} \bar{T}$ and $h=\max _{T \in \mathcal{T}_{h}} h_{T}$, with $h_{T}$ denoting the diameter of $T$. We also denote by $\partial T$ the boundary of a mesh element $T \in \mathcal{T}_{h}$. The set of mesh faces $\mathcal{F}_{h}$ is a finite collection of open disjoint line segments in $\bar{\Omega}$ with nonzero length such that, for all $F \in \mathcal{F}_{h}$, (i) either there exist two 


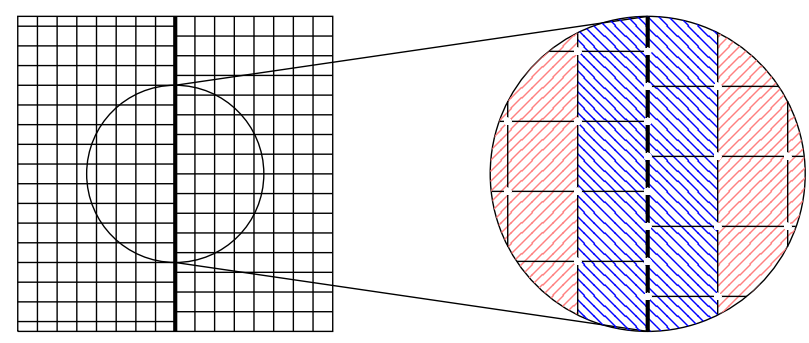

Pentagons

Squares

Figure 2: Treatment of nonconforming fracture discretizations.

distinct mesh elements $T_{1}, T_{2} \in \mathcal{T}_{h}$ such that $F \subset \partial T_{1} \cap \partial T_{2}$ (and $F$ is called an interface) or (ii) there exist a (unique) mesh element $T \in \mathcal{T}_{h}$ such that $F \subset \partial T \cap \partial \Omega$ (and $F$ is called a boundary face). We assume that $\mathcal{F}_{h}$ partitions the mesh skeleton in the sense that $\bigcup_{T \in \mathcal{T}_{h}} \partial T=\bigcup_{F \in \mathcal{F}_{h}} \bar{F}$.

Remark 8 (Mesh faces). Despite working in two space dimensions, we use the terminology "face" over "edge" in order to (i) be consistent with the standard HHO nomenclature and (ii) stress the fact that faces need not coincide with polygonal edges (but can be subsets thereof); see also Remark 9.

We denote by $\mathcal{F}_{h}^{\mathrm{i}}$ the set of all interfaces and by $\mathcal{F}_{h}^{\mathrm{b}}$ the set of all boundary faces, so that $\mathcal{F}_{h}=\mathcal{F}_{h}^{\mathrm{i}} \cup \mathcal{F}_{h}^{\mathrm{b}}$. The length of a face $F \in \mathcal{F}_{h}$ is denoted by $h_{F}$. For any mesh element $T \in \mathcal{T}_{h}, \mathcal{F}_{T}$ is the set of faces that lie on $\partial T$ and, for any $F \in \mathcal{F}_{T}, \boldsymbol{n}_{T F}$ is the unit normal to $F$ pointing out of $T$. Symmetrically, for any $F \in \mathcal{F}_{h}, \mathcal{T}_{F}$ is the set containing the mesh elements sharing the face $F$ (two if $F$ is an interface, one if $F$ is a boundary face).

To account for the presence of the fracture, we make the following assumption.

Assumption 1 (Geometric compliance with the fracture). The mesh is compliant with the fracture, i.e., there exists a subset $\mathcal{F}_{h}^{\Gamma} \subset \mathcal{F}_{h}^{\mathrm{i}}$ such that $\bar{\Gamma}=\bigcup_{F \in \mathcal{F}_{h}^{\Gamma}} \bar{F}$. As a result, $\mathcal{F}_{h}^{\Gamma}$ is a (1-dimensional) mesh of the fracture.

Remark 9 (Polygonal meshes and geometric compliance with the fracture). Fulfilling Assumption 1 does not pose particular problems in the context of polygonal methods, even when the fracture discretization is nonconforming in the classical sense. Consider, e.g., the situation illustrated in Figure 2, where the fracture lies at the intersection of two nonmatching Cartesian submeshes. In this

Referee case, no special treatment is required if the mesh elements in contact with the fracture are treated as pentagons with two coplanar faces instead of rectangles. This is possible since, as already pointed out, the set of mesh faces $\mathcal{F}_{h}$ does not need to coincide with the set of polygonal edges of $\mathcal{T}_{h}$.

The set of vertices of the fracture is denoted by $\mathcal{V}_{h}$ and, for all $F \in \mathcal{F}_{h}^{\Gamma}$, we denote by $\mathcal{V}_{F}$ the vertices of $F$. Symmetrically, for any $V \in \mathcal{V}_{h}, \mathcal{F}_{V}$ is the set containing the fracture faces sharing the vertex $V$ (two if $V$ is an internal vertex, one if $V$ is on the boundary on the fracture). For all $F \in \mathcal{F}_{h}^{\Gamma}$ and all $V \in \mathcal{V}_{F}, \tau_{F V}$ denotes the unit vector tangent to the fracture and oriented so that it points out of $F$ from $V$. Finally, $\mathcal{V}_{h}^{\mathrm{i}}$ is the set containing the internal vertices and $\mathcal{V}_{h}^{\mathrm{b}}$ is the set containing the points in $\partial \Gamma$, so that $\mathcal{V}_{h}=\mathcal{V}_{h}^{\mathrm{i}} \cup \mathcal{V}_{h}^{\mathrm{b}}$.

To avoid dealing with jumps of the problem data inside mesh elements, as well as on boundary and fracture faces, we additionally make the following assumption.

Assumption 2 (Compliance with the problem data). The mesh is compliant with the data, i.e.: (i) for each mesh element $T \in \mathcal{T}_{h}$, there exists a unique sudomain $\omega_{\mathrm{B}} \in \mathcal{P}_{\mathrm{B}}$ (see (2)) such that $T \subset \omega_{\mathrm{B}}$;

(ii) for each fracture face $F \in \mathcal{F}_{h}^{\Gamma}$, there is a unique subdomain $\omega_{\Gamma} \in \mathcal{P}_{\Gamma}$ (see (3)) such that $F \subset \omega_{\Gamma}$. 


\section{The Hybrid High-Order method}

In this section, we formulate the HHO discretization of problems (6) (Darcy flow) and (14) (steady Referee passive transport).

\subsection{Darcy flow}

We start with the discretization of problem (6), which is closely inspired by [15]. Through this section, we denote by $l \geq 0$ a fixed integer polynomial degree.

\subsubsection{Discrete bulk Darcy velocity unkonwns, bulk Darcy velocity reconstruction, and permeability- weighted product of Darcy velocities}

Let an element $T \in \mathcal{T}_{h}$ be fixed, and denote by $\boldsymbol{K}_{T}$ the (constant) restriction to $T$ of the bulk permeability. For any integer $m \geq 0$, set

$$
\boldsymbol{U}_{T}^{m}:=\boldsymbol{K}_{T} \nabla \mathbb{P}^{m}(T)
$$

with $\mathbb{P}^{m}(T)$ denoting the space spanned by the restriction to $T$ of two-variate polynomials of total degree up to $m$. We define the following space of fully discontinuous bulk Darcy velocity unknowns: $\widetilde{\boldsymbol{U}}_{h}^{l}:=\left\{\underline{\boldsymbol{q}}_{h}:=\left(\boldsymbol{q}_{T},\left(q_{T F}\right)_{F \in \mathcal{F}_{T}}\right)_{T \in \mathcal{T}_{h}}:\right.$ for all $T \in \mathcal{T}_{h}, \boldsymbol{q}_{T} \in \boldsymbol{U}_{T}^{l}$ and $q_{T F} \in \mathbb{P}^{l}(F)$ for all $\left.F \in \mathcal{F}_{T}\right\}$.

Referee \#3, typos

For any $T \in \mathcal{T}_{h}$, the element-based unknown $\boldsymbol{q}_{T}$ represents the Darcy velocity inside the element, while the face-based unknown $q_{T F}, F \in \mathcal{F}_{T}$, represents the normal Darcy velocity exiting $T$ through $F$. Furthermore, we denote by $\underline{\boldsymbol{U}}_{T}^{l}$ the restriction of $\underline{\boldsymbol{U}}_{h}^{l}$ to $T$ and, for any $\underline{\boldsymbol{q}}_{h} \in \underline{\widetilde{U}}_{h}^{l}$, we let $\underline{\boldsymbol{q}}_{T}:=\left(\boldsymbol{q}_{T},\left(q_{T F}\right)_{F \in \mathcal{F}_{T}}\right) \in \underline{\boldsymbol{U}}_{T}^{l}$. The following subspace of $\underline{\boldsymbol{U}}_{h}^{l}$ strongly incorporates the continuity of Darcy velocity unknowns at each interface $F \in \mathcal{F}_{h}^{\mathrm{i}} \backslash \mathcal{F}_{h}^{\Gamma}$ contained in the bulk region, as well as the homogeneous Neumann boundary condition on $\partial \Omega_{\mathrm{B}}$ :

$$
\underline{\boldsymbol{U}}_{h}^{l}:=\left\{\underline{\boldsymbol{q}}_{h} \in \underline{\boldsymbol{U}}_{h}^{l}: \llbracket \underline{\boldsymbol{q}}_{h} \rrbracket_{F}=0 \text { for all } F \in \mathcal{F}_{h}^{\mathrm{i}} \backslash \mathcal{F}_{h}^{\Gamma} \text { and } q_{F}=0 \text { for all } F \in \mathcal{F}_{h}^{\mathrm{b}}\right\},
$$

where, for all $F \in \mathcal{F}_{h}^{\text {b }}$, we have set $q_{F}:=q_{T F}$ with $T$ denoting the unique mesh element such that $F \in \mathcal{F}_{T}$ and, for all $F \in \mathcal{F}_{h}^{\mathrm{i}}$, we have defined the jump operator such that, for any $\underline{\boldsymbol{q}}_{h} \in \underline{\boldsymbol{U}}_{h}^{l}$,

$$
\llbracket \underline{\boldsymbol{q}}_{h} \rrbracket_{F}:=\sum_{T \in \mathcal{T}_{F}} q_{T F} .
$$

For all $T \in \mathcal{T}_{h}$, we define the local discrete Darcy velocity reconstruction operator $\boldsymbol{F}_{T}^{l+1}: \underline{U}_{T}^{l} \rightarrow$ $\boldsymbol{U}_{T}^{l+1}$ (see (22)) such that, for all $\underline{\boldsymbol{q}}_{T}=\left(\boldsymbol{q}_{T},\left(q_{T F}\right)_{F \in \mathcal{F}_{T}}\right) \in \underline{\boldsymbol{U}}_{T}^{l}, \boldsymbol{F}_{T}^{l+1} \underline{\boldsymbol{q}}_{T}$ solves

$$
\int_{T} \boldsymbol{F}_{T}^{l+1} \underline{\boldsymbol{q}}_{T} \cdot \nabla w_{T}=\int_{T} \boldsymbol{q}_{T} \cdot \nabla \pi_{T}^{l} w_{T}+\sum_{F \in \mathcal{F}_{T}} \int_{F} q_{T F}\left(w_{T}-\pi_{T}^{l} w_{T}\right) \quad \forall w_{T} \in \mathbb{P}^{l+1}(T),
$$

with $\pi_{T}^{l}: L^{1}(T) \rightarrow \mathbb{P}^{l}(T)$ denoting the $L^{2}$-orthogonal projector on $\mathbb{P}^{l}(T)$; see, e.g., [19, Appendix A.2]. Notice that the quantity $\boldsymbol{F}_{T}^{l+1} \underline{\boldsymbol{q}}_{T}$ provides a representation of the Darcy velocity inside $T$ one degree higher than the element-based unknown $\boldsymbol{q}_{T}$. It can be checked that condition (24) defines a unique element of $\boldsymbol{U}_{T}^{l+1}$, and that it is equivalent to [15, Eq. (19)] with discrete divergence operator expanded according to its definition.
Referee \#3, typos
Referee \#2, comment 2 
Based on this Darcy velocity reconstruction operator, we define the global permeability-weighted product of Darcy velocities $a_{K, h}^{l}: \underline{\boldsymbol{U}}_{h}^{l} \times \underline{\boldsymbol{U}}_{h}^{l} \rightarrow \mathbb{R}$ such that, for all $\left.\underline{\boldsymbol{u}}_{h}, \underline{\boldsymbol{q}}_{h}\right) \in \underline{\boldsymbol{U}}_{h}^{l} \times \underline{\boldsymbol{U}}_{h}^{l}$,

$$
a_{K, h}^{l}\left(\underline{\boldsymbol{u}}_{h}, \underline{\boldsymbol{q}}_{h}\right):=\sum_{T \in \mathcal{T}_{h}}\left(\int_{T} \boldsymbol{K}_{T}^{-1} \boldsymbol{F}_{T}^{l+1} \underline{\boldsymbol{u}}_{T} \cdot \boldsymbol{F}_{T}^{l+1} \underline{\boldsymbol{q}}_{T}+s_{K, T}^{l}\left(\underline{\boldsymbol{u}}_{T}, \underline{\boldsymbol{q}}_{T}\right)\right) .
$$

Here, the first term is the Galerkin contribution responsible for consistency while, for all $T \in \mathcal{T}_{h}$, $s_{K, T}^{l}: \underline{\boldsymbol{U}}_{T}^{l} \times \underline{\boldsymbol{U}}_{T}^{l} \rightarrow \mathbb{R}$ is the stabilization bilinear form such that, for all $\left(\underline{\boldsymbol{u}}_{T}, \underline{\boldsymbol{q}}_{T}\right) \in \underline{\boldsymbol{U}}_{T}^{l} \times \underline{\boldsymbol{U}}_{T}^{l}$,

$$
s_{K, T}^{l}\left(\underline{\boldsymbol{u}}_{T}, \underline{\boldsymbol{q}}_{T}\right):=\sum_{F \in \mathcal{F}_{T}} \int_{F} \frac{h_{F}}{K_{T F}}\left(\boldsymbol{F}_{T}^{l+1} \underline{\boldsymbol{u}}_{T} \cdot \boldsymbol{n}_{T F}-u_{T F}\right)\left(\boldsymbol{F}_{T}^{l+1} \underline{\boldsymbol{q}}_{T} \cdot \boldsymbol{n}_{T F}-q_{T F}\right),
$$

where, for all $F \in \mathcal{F}_{T}$, we have set $K_{T F}:=\boldsymbol{K}_{T} \boldsymbol{n}_{T F} \cdot \boldsymbol{n}_{T F}$.

\subsubsection{Discrete fracture pressure unknowns, fracture pressure reconstruction, and tangential diffusion bilinear form}

The space of discrete fracture pressure unknowns is given by

$$
\underline{P}_{\Gamma, h}^{l}:=\left\{\underline{z}_{h}^{\Gamma}:=\left(\left(z_{F}^{\Gamma}\right)_{F \in \mathcal{F}_{h}^{\Gamma}},\left(z_{V}^{\Gamma}\right)_{V \in \mathcal{V}_{h}}\right): z_{F}^{\Gamma} \in \mathbb{P}^{l}(F) \text { for all } F \in \mathcal{F}_{h}^{\Gamma} \text { and } z_{V}^{\Gamma} \in \mathbb{R} \text { for all } V \in \mathcal{V}_{h}\right\} .
$$

For all $F \in \mathcal{F}_{h}^{\Gamma}$, we denote by $\underline{P}_{\Gamma, F}^{l}$ the restriction of $\underline{P}_{\Gamma, h}^{l}$ to $F$, and set $\underline{z}_{F}^{\Gamma}:=\left(z_{F}^{\Gamma},\left(z_{V}^{\Gamma}\right)_{V \in \mathcal{V}_{F}}\right) \in \underline{P}_{\Gamma, F}^{l}$. We also introduce the following subspace which embeds the zero-mean value constraint:

$$
\underline{P}_{\Gamma, h, 0}^{l}:=\left\{\underline{z}_{h}^{\Gamma} \in \underline{P}_{\Gamma, h}^{l}: \int_{\Gamma} z_{h}^{\Gamma}=0\right\},
$$

where $z_{h}^{\Gamma} \in \mathbb{P}^{l}\left(\mathcal{F}_{h}^{\Gamma}\right)$ is the broken polynomial function on $\mathcal{F}_{h}^{\Gamma}$ such that $\left(z_{h}^{\Gamma}\right)_{\mid F}:=z_{F}^{\Gamma}$ for all $F \in \mathcal{F}_{h}^{\Gamma}$.

Let $F \in \mathcal{F}_{h}^{\Gamma}$ and denote by $K_{F}$ the (constant) restriction to $F$ of the fracture permeability. We define the local fracture pressure reconstruction operator $r_{K, F}^{l+1}: \underline{P}_{\Gamma, F}^{l} \rightarrow \mathbb{P}^{l+1}(F)$ such that, for all $\underline{z}_{F}^{\Gamma}=\left(z_{F}^{\Gamma},\left(z_{V}^{\Gamma}\right)_{\mathcal{V}_{F}}\right) \in \underline{P}_{\Gamma, F}^{l}, r_{K, F}^{l+1} \underline{z}_{F}^{\Gamma}$ is such that, for all $w_{F}^{\Gamma} \in \mathbb{P}^{l+1}(F)$,

$$
\int_{F} K_{F} \nabla_{\tau} r_{K, F}^{l+1} \underline{z}_{F}^{\Gamma} \cdot \nabla_{\tau} w_{F}^{\Gamma}=-\int_{F}\left(z_{F}^{\Gamma} \nabla_{\tau} \cdot\left(K_{F} \nabla_{\tau} w_{F}^{\Gamma}\right)\right)+\sum_{V \in \mathcal{V}_{F}} z_{V}^{\Gamma}\left(K_{F} \nabla_{\tau} w_{F}^{\Gamma}\right)(V) \cdot \tau_{F V}
$$

This relation defines a unique element $\nabla_{\tau} r_{K, F}^{l+1} \underline{z}_{F}^{\Gamma}$, hence a polynomial $r_{K, F}^{l+1} \underline{z}_{F}^{\Gamma} \in \mathbb{P}^{l+1}(F)$ up to an additive constant, which we fix by additionally imposing that

$$
\int_{F}\left(r_{K, F}^{l+1} \underline{z}_{F}^{\Gamma}-z_{F}^{\Gamma}\right)=0
$$

The reconstruction $r_{K, F}^{l+1} z_{F}^{\Gamma}$ provides inside $F$ a representation of the fracture pressure one degree higher than the element-based fracture unknown $z_{F}^{\Gamma}$.

We can now define the tangential diffusion bilinear form $a_{K, h}^{\Gamma, l}: \underline{P}_{\Gamma, h}^{l} \times \underline{P}_{\Gamma, h}^{l} \rightarrow \mathbb{R}$ such that

$$
a_{K, h}^{\Gamma, l}\left(\underline{z}_{h}^{\Gamma}, \underline{q}_{h}^{\Gamma}\right):=\sum_{F \in \mathcal{F}_{h}^{\Gamma}}\left(\int_{F} K_{F} \nabla_{\tau} r_{K, F}^{l+1} \underline{z}_{F}^{\Gamma} \cdot \nabla_{\tau} r_{K, F}^{l+1} \underline{q}_{F}^{\Gamma}+s_{K, F}^{\Gamma, l}\left(\underline{z}_{F}^{\Gamma}, \underline{q}_{F}^{\Gamma}\right)\right),
$$

where the first term is the Galerkin contribution responsible for consistency, while $s_{K, F}^{\Gamma, l}: \underline{P}_{\Gamma, F}^{l} \times$ $\underline{P}_{\Gamma, F}^{l} \rightarrow \mathbb{R}$ is the stabilization bilinear form such that, for all $\left(\underline{z}_{F}^{\Gamma}, \underline{q}_{F}^{\Gamma}\right) \in \underline{P}_{\Gamma, F}^{l} \times \underline{P}_{\Gamma, F}^{l}$,

$$
s_{K, F}^{\Gamma, l}\left(\underline{z}_{F}^{\Gamma}, \underline{q}_{F}^{\Gamma}\right):=\sum_{V \in \mathcal{V}_{F}} \frac{K_{F}}{h_{F}}\left(R_{K, F}^{l+1} \underline{z}_{F}^{\Gamma}(V)-z_{V}^{\Gamma}\right)\left(R_{K, F}^{l+1} \underline{q}_{F}^{\Gamma}(V)-q_{V}^{\Gamma}\right),
$$

with $R_{K, F}^{l+1}: \underline{P}_{\Gamma, F}^{l} \rightarrow \mathbb{P}^{l+1}(F)$ such that, for all $\underline{z}_{F}^{\Gamma} \in \underline{P}_{\Gamma, F}^{l}, R_{K, F}^{l+1} \underline{z}_{F}^{\Gamma}:=z_{F}^{\Gamma}+\left(r_{K, F}^{l+1} \underline{z}_{F}^{\Gamma}-\pi_{F}^{l} r_{K, F}^{l+1} \underline{z}_{F}^{\Gamma}\right)$. 


\subsubsection{Discrete flow problem}

Let an integer $k \geq 0$ be fixed. Following [3], in order to have a sufficiently accurate representation of the Darcy velocity when writing the HHO approximation of degree $k$ of the transport problem (14), we solve the flow problem (6) with an HHO approximation of degree $2 k$. Thus, the bulk velocity, bulk pressure, and fracture pressure will be sought, respectively, in $\underline{U}_{h}^{2 k}($ see $(23)), P_{\mathrm{B}, h}^{2 k}:=\mathbb{P}^{2 k}\left(\mathcal{T}_{h}\right)$ (the space of broken polynomials of total degree $\leq 2 k$ over $\mathcal{T}_{h}$ ), and $\underline{P}_{\Gamma, h}^{2 k}$ (see (27)). The discrete counterparts of the continuous bilinear forms defined in Section 2.2 are the bilinear forms $a_{K, h}^{\xi, 2 k}$ : $\underline{\boldsymbol{U}}_{h}^{2 k} \times \underline{\boldsymbol{U}}_{h}^{2 k} \rightarrow \mathbb{R}, b_{h}^{2 k}: \underline{\boldsymbol{U}}_{h}^{2 k} \times P_{\mathrm{B}, h}^{2 k} \rightarrow \mathbb{R}, d_{h}^{2 k}: \underline{\boldsymbol{U}}_{h}^{2 k} \times \underline{P}_{\Gamma, h}^{2 k} \rightarrow \mathbb{R}$ such that

$$
\begin{aligned}
a_{K, h}^{\xi, 2 k}\left(\underline{\boldsymbol{u}}_{h}, \underline{\boldsymbol{q}}_{h}\right) & :=a_{K, h}^{2 k}\left(\underline{\boldsymbol{u}}_{h}, \underline{\boldsymbol{q}}_{h}\right)+\sum_{F \in \mathcal{F}_{h}^{\Gamma}}\left(\left(\lambda_{F}^{\xi} \llbracket \underline{\boldsymbol{u}}_{h} \rrbracket_{F}, \llbracket \underline{\boldsymbol{q}}_{h} \rrbracket_{F}\right)_{F}+\left(\lambda_{F}\left\{\left\{\underline{\boldsymbol{u}}_{h}\right\}\right\}_{F},\left\{\left\{\underline{\boldsymbol{q}}_{h}\right\}\right\}_{F}\right)_{F}\right), \\
b_{h}^{2 k}\left(\underline{\boldsymbol{u}}_{h}, p_{h}\right) & :=\sum_{T \in \mathcal{T}_{h}}\left(-\int_{T} \boldsymbol{u}_{T} \cdot \nabla p_{T}+\sum_{F \in \mathcal{F}_{T}} \int_{F} u_{T F} p_{T}\right), \\
d_{h}^{2 k}\left(\underline{\boldsymbol{u}}_{h}, \underline{p}_{h}^{\Gamma}\right) & :=\sum_{F \in \mathcal{F}_{h}^{\Gamma}} \int_{F} \llbracket \underline{\boldsymbol{u}}_{h} \rrbracket_{F} p_{F}^{\Gamma},
\end{aligned}
$$

where the bilinear forms $a_{K, h}^{2 k}$ and $a_{K, h}^{\Gamma, 2 k}$ are defined by (25) and (29), respectively, and, for all $p_{h} \in P_{\mathrm{B}, h}^{2 k}$ and all $T \in \mathcal{T}_{h}$, we have set $p_{T}:=p_{h \mid T}$.

Letting $\mathcal{A}_{\xi, h, 2 k}^{\text {flow }}:\left(\underline{\boldsymbol{U}}_{h}^{2 k} \times P_{\mathrm{B}, h}^{2 k} \times \underline{P}_{\Gamma, h}^{2 k}\right) \times\left(\underline{\boldsymbol{U}}_{h}^{2 k} \times P_{\mathrm{B}, h}^{2 k} \times \underline{P}_{\Gamma, h}^{2 k}\right) \rightarrow \mathbb{R}$ be the global bilinear form such that

$$
\begin{aligned}
\mathcal{A}_{\xi, h, 2 k}^{\text {flow }}\left(\left(\underline{\boldsymbol{u}}_{h}, p_{h}, \underline{p}_{h}^{\Gamma}\right),\left(\underline{\boldsymbol{q}}_{h}, z_{h}, \underline{z}_{h}^{\Gamma}\right)\right):=a_{K, h}^{\xi, 2 k}\left(\underline{\boldsymbol{u}}_{h}, \underline{\boldsymbol{q}}_{h}\right)+ & b_{h}^{2 k}\left(\underline{\boldsymbol{u}}_{h}, z_{h}\right)-b_{h}^{2 k}\left(\underline{\boldsymbol{q}}_{h}, p_{h}\right) \\
& +d_{h}^{2 k}\left(\underline{\boldsymbol{q}}_{h}, \underline{p}_{h}^{\Gamma}\right)-d_{h}^{2 k}\left(\underline{\boldsymbol{u}}_{h}, \underline{z}_{h}^{\Gamma}\right)+a_{K, h}^{\Gamma, 2 k}\left(\underline{p}_{h}^{\Gamma}, \underline{z}_{h}^{\Gamma}\right),
\end{aligned}
$$

the HHO discretization of problem (6) reads: Find $\left(\underline{\boldsymbol{u}}_{h}, p_{h}, \underline{p}_{h}^{\Gamma}\right) \in \underline{\boldsymbol{U}}_{h}^{2 k} \times P_{\mathrm{B}, h}^{2 k} \times \underline{P}_{\Gamma, h, 0}^{2 k}$ such that, for all $\left(\underline{\boldsymbol{q}}_{h}, z_{h}, \underline{z}_{h}^{\Gamma}\right) \in \underline{\boldsymbol{U}}_{h}^{2 k} \times P_{\mathrm{B}, h}^{2 k} \times \underline{P}_{\Gamma, h}^{2 k}$,

$$
\mathcal{A}_{\xi, h, 2 k}^{\text {flow }}\left(\left(\underline{\boldsymbol{u}}_{h}, p_{h}, \underline{p}_{h}^{\Gamma}\right),\left(\underline{\boldsymbol{q}}_{h}, z_{h}, \underline{z}_{h}^{\Gamma}\right)\right)=\left(f, z_{h}\right)_{\Omega_{\mathrm{B}}}+\left(\ell_{\Gamma} f_{\Gamma}, z_{h}^{\Gamma}\right)_{\Gamma} .
$$

\subsection{Passive transport}

We now formulate the HHO discretization of the steady passive transport problem (14). In what follows, the polynomial degree $k$ is the same as in Section 4.1.3.

Referee \# 3, comment

\subsubsection{Discrete bulk concentration unknowns, bulk concentration reconstruction, and molec- ular diffusion bilinear form}

We define the fully discontinuous space of bulk concentration unknowns as follows:

$\underline{P}_{\mathrm{B}, h}^{k}:=\left\{\underline{z}_{h}=\left(z_{T},\left(z_{T F}\right)_{F \in \mathcal{F}_{T}}\right)_{T \in \mathcal{T}_{h}}:\right.$ for all $T \in \mathcal{T}_{h}, z_{T} \in \mathbb{P}^{k}(T)$ and $z_{T F} \in \mathbb{P}^{k}(F)$ for all $\left.F \in \mathcal{F}_{T}\right\}$.

For all $T \in \mathcal{T}_{h}$, we denote by $\underline{P}_{\mathrm{B}, T}^{k}$ the restriction of $\widetilde{P}_{\mathrm{B}, h}^{k}$ to $T$, and we set $\underline{z}_{T}=\left(z_{T},\left(z_{T F}\right)_{F \in \mathcal{F}_{T}}\right) \in \underline{P}_{\mathrm{B}, T}^{k}$. For any interface $F \in \mathcal{F}_{h}^{\mathrm{i}}$ shared by distinct elements $T_{1}, T_{2} \in \mathcal{T}_{F}$, we introduce the jump and average operators such that, for any $\underline{z}_{h} \in \widetilde{P}_{\mathrm{B}, h}^{k}$,

$$
\llbracket \underline{z}_{h} \rrbracket_{F}=z_{T_{1} F}-z_{T_{2} F}, \quad\left\{\left\{\underline{z}_{h}\right\}_{F}=\frac{z_{T_{1} F}+z_{T_{2} F}}{2} .\right.
$$


The following subspace of $\widetilde{P}_{\mathrm{B}, h}^{k}$ strongly incorporates the continuity of concentration unknowns across interfaces contained in the bulk region:

$$
\underline{P}_{\mathrm{B}, h}^{k}:=\left\{\underline{z}_{h} \in \widehat{P}_{\mathrm{B}, h}^{k}: \llbracket \underline{z}_{h} \rrbracket_{F}=0 \text { for all } F \in \mathcal{F}_{h}^{\mathrm{i}} \backslash \mathcal{F}_{h}^{\Gamma}\right\} .
$$

Let now an element $T \in \mathcal{T}_{h}$ be fixed, and denote by $\boldsymbol{D}_{T}$ the restriction to $T$ of the bulk diffusiondispersion tensor. We define the bulk concentration reconstruction operator $r_{D, T}^{k+1}: \underline{P}_{\mathrm{B}, T}^{k} \rightarrow \mathbb{P}^{k+1}(T)$ such that, for all $\underline{z}_{T}=\left(z_{T},\left(z_{T F}\right)_{F \in \mathcal{F}_{t}}\right) \in \underline{P}_{\mathrm{B}, T}^{k}, r_{D, T}^{k+1} \underline{z}_{T}$ solves

$$
\int_{T} \boldsymbol{D}_{T} \nabla r_{D, T}^{k+1} \underline{z}_{T} \cdot \nabla w_{T}=-\int_{T}\left(z_{T} \nabla \cdot\left(\boldsymbol{D}_{T} \nabla w_{T}\right)\right)+\sum_{F \in \mathcal{F}_{T}} \int_{F} z_{T F}\left(\boldsymbol{D}_{T} \nabla w_{T} \cdot \boldsymbol{n}_{T F}\right) \quad \forall w_{T} \in \mathbb{P}^{k+1}(T) .
$$

Referee \#1, comment 5

This condition defines $r_{D, T}^{k+1} \underline{z}_{T}$ up to a constant, which we fix by additionally imposing that

$$
\int_{T}\left(r_{D, T}^{k+1} \underline{z}_{T}-z_{T}\right)=0
$$

The polynomial $r_{D, T}^{k+1} \underline{z}_{T}$ provides a representation of the concentration inside $T$ one degree higher than the element-based unknown $z_{T}$.

We are now ready to define a global molecular diffusion bilinear form closely inspired by [21]. More precisely, we let $a_{D, h}^{k}: \underline{P}_{\mathrm{B}, h}^{k} \times \underline{P}_{\mathrm{B}, h}^{k} \rightarrow \mathbb{R}$ be such that, for all $\left(\underline{c}_{h}, \underline{z}_{h}\right) \in \underline{P}_{\mathrm{B}, h}^{k} \times \underline{P}_{\mathrm{B}, h}^{k}$,

$$
a_{D, h}^{k}\left(\underline{c}_{h}, \underline{z}_{h}\right):=\sum_{T \in \mathcal{T}_{h}}\left(\int_{T} \boldsymbol{D}_{T} \nabla r_{D, T}^{k+1} \underline{c}_{T} \cdot \nabla r_{D, T}^{k+1} \underline{z}_{T}+s_{D, T}^{k}\left(\underline{c}_{T}, \underline{z}_{T}\right)\right)
$$

where the first term is the Galerkin contribution responsible for consistency, while $s_{D, T}^{k}: \underline{P}_{\mathrm{B}, T}^{k} \times$ $\underline{P}_{\mathrm{B}, T}^{k} \rightarrow \mathbb{R}$ is the stabilization bilinear form such that, for all $\left(\underline{c}_{T}, \underline{z}_{T}\right) \in \underline{P}_{\mathrm{B}, T}^{k} \times \underline{P}_{\mathrm{B}, T}^{k}$,

$$
s_{D, T}^{k}\left(\underline{c}_{T}, \underline{z}_{T}\right):=\sum_{F \in \mathcal{F}_{T}} \int_{F} \frac{D_{T F}}{h_{F}}\left(R_{D, T}^{k+1} \underline{c}_{T}-c_{T F}\right)\left(R_{D, T}^{k+1} \underline{z}_{T}-z_{T F}\right),
$$

with $D_{T F}:=\boldsymbol{D}_{T} \boldsymbol{n}_{T F} \cdot \boldsymbol{n}_{T F}$ for all $F \in \mathcal{F}_{T}$ and $R_{D, T}^{k+1}: \underline{P}_{\mathrm{B}, T}^{k} \rightarrow \mathbb{P}^{k+1}(T)$ is such that, for all $\underline{z}_{T} \in \underline{P}_{\mathrm{B}, T}^{k}$, $R_{D, T}^{k+1} \underline{z}_{T}:=z_{T}+\left(r_{D, T}^{k+1} \underline{z}_{T}-\pi_{T}^{k} r_{D, T}^{k+1} \underline{z}_{T}\right)$.

\subsubsection{Fracture concentration unknowns, fracture concentration reconstruction, and molecu- lar diffusion bilinear form}

The fracture concentration is sought in the space $\underline{P}_{\Gamma, h}^{k}$ defined by (26) with $l=k$. For all $F \in \mathcal{F}_{h}^{\Gamma}$, we define the fracture concentration reconstruction operator $r_{D, F}^{k+1}: \underline{P}_{\Gamma, F}^{k} \rightarrow \mathbb{P}^{k+1}(F)$ as in (28) setting $l=k$ and replacing $K_{F}$ by $D_{F}:=D_{\Gamma \mid F}$. Similary, we denote by $a_{D, h}^{\Gamma, k}: \underline{P}_{\Gamma, h}^{k} \times \underline{P}_{\Gamma, h}^{k} \rightarrow \mathbb{R}$ the tangential molecular diffusion bilinear form defined as (29)-(30) with $l=k$ and $K_{F}$ replaced by $D_{F}$.

\subsubsection{Darcy velocities and advection-reaction bilinear forms in the bulk region and in the fracture}

In order to discretize the advection-reaction terms that appear in the passive transport problem, we need suitable representations of the Darcy velocity both in the bulk region and inside the fracture. 
Denote by $\left(\underline{\boldsymbol{u}}_{h}, p_{h}, \underline{p}_{h}^{\Gamma}\right) \in \underline{\boldsymbol{U}}_{h}^{2 k} \times P_{\mathrm{B}, h}^{2 k} \times \underline{P}_{\Gamma, h, 0}^{2 k}$ the solution of the discrete flow problem (31). For any $T \in \mathcal{T}_{h}$, taking in (31) $\underline{\boldsymbol{q}}_{h}=\underline{\mathbf{0}}, z_{h}$ such that $z_{T^{\prime}}=0$ for all $T^{\prime} \in \mathcal{T}_{h} \backslash\{T\}$ while $z_{T}$ spans $\mathbb{P}^{2 k}(T)$, and $\underline{z}_{h}^{\Gamma}=\underline{0}$, we infer the following local balance for the discrete bulk Darcy velocity:

$$
\int_{T}-\boldsymbol{u}_{T} \cdot \nabla z_{T}+\sum_{F \in \mathcal{F}_{T}} \int_{F} u_{T F} z_{T}=\int_{T} f z_{T} \quad \forall z_{T} \in \mathbb{P}^{2 k}(T)
$$

Additionally, by definition (23) of $\underline{\boldsymbol{U}}_{h}^{2 k}$, the Darcy velocity thus defined has continuous normal components across interfaces contained in the bulk in the sense that $\llbracket \underline{u}_{h} \rrbracket_{F}=0$ for all $F \in \mathcal{F}_{h}^{\mathrm{i}} \backslash \mathcal{F}_{h}^{\Gamma}$ where the jump operator is defined applying (32) componentwise. Thus, $\underline{\boldsymbol{u}}_{h}$ is the natural candidate to play the role of the Darcy velocity in the bulk region.

Let now a fracture face $F \in \mathcal{F}_{h}^{\Gamma}$ be fixed, and define the fracture Darcy velocity $\underline{\boldsymbol{u}}_{F}^{\Gamma}=$ $\left(\boldsymbol{u}_{F}^{\Gamma},\left(u_{F V}^{\Gamma}\right)_{V \in \mathcal{V}_{F}}\right)$ such that

$$
\boldsymbol{u}_{F}^{\Gamma}:=-K_{F} \nabla_{\tau} r_{K, F}^{2 k+1} \underline{p}_{F}^{\Gamma} \text { and, for all } V \in \mathcal{V}_{F}, u_{F V}^{\Gamma}:= \begin{cases}\boldsymbol{u}_{F}^{\Gamma}(V) \cdot \boldsymbol{\tau}_{F V}+\gamma_{F V}^{\mathrm{num}}\left(\underline{p}_{F}^{\Gamma}\right) & \text { if } V \in \mathcal{V}_{h}^{\mathrm{i}} \\ 0 & \text { if } V \in \mathcal{V}_{h}^{\mathrm{b}}\end{cases}
$$

where, for all $V \in \mathcal{V}_{F}, \gamma_{F V}^{\text {num }}: \underline{P}_{\Gamma, F}^{2 k} \rightarrow \mathbb{R}$ is the boundary residual operator defined as in [22, Lemma 3]. With this choice for the fracture Darcy velocity, the following local balance holds for all $F \in \mathcal{F}_{h}^{\Gamma}$ :

$$
-\int_{F} \boldsymbol{u}_{F}^{\Gamma} \cdot \nabla_{\tau} z_{F}^{\Gamma}+\sum_{V \in \mathcal{V}_{F}} u_{F V}^{\Gamma}\left(z_{F}^{\Gamma}(V)-z_{V}^{\Gamma}\right)=\int_{F}\left(\ell_{\Gamma} f_{\Gamma}+\llbracket \underline{\boldsymbol{u}}_{h} \rrbracket_{F}\right) z_{F}^{\Gamma} \quad \forall \underline{z}_{F}^{\Gamma} \in \underline{P}_{\Gamma, F}^{2 k} .
$$

Moreover, the discrete fracture Darcy velocity is continuous across internal vertices, that is to say,

$$
\sum_{F \in \mathcal{F}_{V}} u_{F V}^{\Gamma}=0 \text { for all } V \in \mathcal{V}_{h}^{\mathrm{i}}
$$

$\underline{\boldsymbol{u}}_{F}^{\Gamma}$ is therefore the natural candidate to play the role of the Darcy velocity inside the fracture.

We now have all the ingredients to define discrete counterparts of the advective terms in the bulk region and inside the fracture. More precisely, closely following [20], we define the advection-reaction bilinear forms $a_{\boldsymbol{u}, f, h}^{k}: \underline{P}_{\mathrm{B}, h}^{k} \times \underline{P}_{\mathrm{B}, h}^{k} \rightarrow \mathbb{R}$ and $a_{\boldsymbol{u}, f, h}^{\Gamma, k}: \underline{P}_{\Gamma, h}^{k} \times \underline{P}_{\Gamma, h}^{k}$ such that

$$
\begin{array}{r}
a_{\boldsymbol{u}, f, h}^{k}\left(\underline{c}_{h}, \underline{z}_{h}\right):=\sum_{T \in \mathcal{T}_{h}}\left(\int_{T} c_{T}\left(-\boldsymbol{u}_{T} \cdot \nabla z_{T}+f^{-} z_{T}\right)+\sum_{F \in \mathcal{F}_{T}} \int_{F} u_{T F} c_{T}\left(z_{T}-z_{T F}\right)+s_{\boldsymbol{u}, T}^{k}\left(\underline{c}_{T}, \underline{z}_{T}\right)\right), \\
a_{\boldsymbol{u}, f, h}^{\Gamma, k}\left(\underline{c}_{h}^{\Gamma}, \underline{z}_{h}^{\Gamma}\right):=\sum_{F \in \mathcal{F}_{h}^{\Gamma}}\left(\int_{F} c_{F}^{\Gamma}\left(-\boldsymbol{u}_{F}^{\Gamma} \cdot \nabla_{\tau} z_{F}^{\Gamma}+\ell_{\Gamma} f_{\Gamma}^{-} z_{F}^{\Gamma}\right)+\sum_{V \in \mathcal{V}_{F}} u_{F V}^{\Gamma} c_{F}^{\Gamma}(V)\left(z_{F}^{\Gamma}(V)-z_{V}^{\Gamma}\right)+s_{\boldsymbol{u}, F}^{\Gamma, k}\left(\underline{c}_{F}^{\Gamma}, \underline{z}_{F}^{\Gamma}\right)\right),
\end{array}
$$

where, for all $T \in \mathcal{T}_{h}$ and all $F \in \mathcal{F}_{h}^{\Gamma}, s_{\boldsymbol{u}, T}^{k}: \underline{P}_{\mathrm{B}, T}^{k} \times \underline{P}_{\mathrm{B}, T}^{k} \rightarrow \mathbb{R}$ and $s_{\boldsymbol{u}, F}^{\Gamma, k}: \underline{P}_{\Gamma, F}^{k} \times \underline{P}_{\Gamma, F}^{k} \rightarrow \mathbb{R}$ are the upwind stabilization bilinear forms respectively in the bulk and inside the fracture such that

$$
\begin{aligned}
s_{\boldsymbol{u}, T}^{k}\left(\underline{c}_{T}, \underline{z}_{T}\right) & :=\sum_{F \in \mathcal{F}_{T}} \int_{F} \frac{\left|u_{T F}\right|-u_{T F}}{2}\left(c_{T}-c_{T F}\right)\left(z_{T}-z_{T F}\right), \\
s_{\boldsymbol{u}, F}^{\Gamma, k}\left(\underline{c}_{F}^{\Gamma}, \underline{z}_{F}^{\Gamma}\right) & :=\sum_{V \in \mathcal{V}_{F}} \frac{\left|u_{F V}^{\Gamma}\right|-u_{F V}^{\Gamma}}{2}\left(c_{F}^{\Gamma}(V)-c_{V}^{\Gamma}\right)\left(z_{F}^{\Gamma}(V)-z_{V}^{\Gamma}\right) .
\end{aligned}
$$




\subsubsection{Passive transport problem}

We are now ready to state the HHO discretization of the steady transport problem (14). At the discrete level, the counterpart of the continuous bilinear form defined in (13) is the bilinear form $\mathcal{A}_{\xi, h, k}^{\text {transp }}:\left(\underline{P}_{\mathrm{B}, h}^{k} \times \underline{P}_{\Gamma, h}^{k}\right) \times\left(\underline{P}_{\mathrm{B}, h}^{k} \times \underline{P}_{\Gamma, h}^{k}\right) \rightarrow \mathbb{R}$ such that

Referee \#3,

comment

$$
\begin{aligned}
\mathcal{A}_{\xi, h, k}^{\text {transp }}\left(\left(\underline{c}_{h}, \underline{c}_{h}^{\Gamma}\right),\left(\underline{z}_{h}, \underline{z}_{F}^{\Gamma}\right)\right):= & a_{D, h}^{k}\left(\underline{c}_{h}, \underline{z}_{h}\right)+a_{\boldsymbol{u}, f, h}^{k}\left(\underline{c}_{h}, \underline{z}_{h}\right)+a_{D, h}^{\Gamma, k}\left(\underline{c}_{h}^{\Gamma}, \underline{z}_{h}^{\Gamma}\right)+a_{\boldsymbol{u}, f, h}^{\Gamma, k}\left(\underline{c}_{h}^{\Gamma}, \underline{z}_{h}^{\Gamma}\right) \\
& +\sum_{F \in \mathcal{F}_{h}^{\Gamma}} \int_{F}\left(\beta_{F}^{\xi}\left(\left\{\left\{\underline{c}_{h}\right\}\right\}_{F}-c_{F}^{\Gamma}\right)\left(\left\{\left\{\underline{z}_{h}\right\}\right\}_{F}-z_{F}^{\Gamma}\right)+\beta_{F} \llbracket \underline{c}_{h} \rrbracket_{F} \llbracket \underline{z}_{h} \rrbracket_{F}\right) \\
& +\sum_{F \in \mathcal{F}_{h}^{\Gamma}} \int_{F}\left(\left\{\left\{\underline{\boldsymbol{u}}_{h}\right\}\right\}_{F}\left\{\left\{\underline{c}_{h}\right\}\right\}_{F} \llbracket \underline{z}_{h} \rrbracket_{F}+\frac{1}{8} \llbracket \underline{\boldsymbol{u}}_{h} \rrbracket_{F} \llbracket \underline{c}_{h} \rrbracket_{F} \llbracket \underline{z}_{h} \rrbracket_{F}\right) \\
& +\sum_{F \in \mathcal{F}_{h}^{\Gamma}} \int_{F} \frac{1}{2} \llbracket \underline{\boldsymbol{u}}_{h} \rrbracket_{F}\left(\left\{\left\{\underline{c}_{h}\right\}\right\}_{F}+c_{F}^{\Gamma}\right)\left(\left\{\left\{\underline{z}_{h}\right\}\right\}_{F}-z_{F}^{\Gamma}\right),
\end{aligned}
$$

where the role of the terms in the last three lines is to enforce the transmission conditions (9) on $\Gamma$. The HHO discretization of problem (14) then reads: Find $\left(\underline{c}_{h}, \underline{c}_{h}^{\Gamma}\right) \in \underline{P}_{\mathrm{B}, h}^{k} \times \underline{P}_{\Gamma, h}^{k}$ such that

Referee \#3, typos

$$
\mathcal{A}_{\xi, h, k}^{\text {transp }}\left(\left(\underline{c}_{h}, \underline{c}_{h}^{\Gamma}\right),\left(\underline{z}_{h}, \underline{z}_{F}^{\Gamma}\right)\right)=\int_{\Omega_{\mathrm{B}}} f^{+} \widehat{c}_{z h}+\int_{\Gamma} \ell_{\Gamma} f_{\Gamma}^{+} \widehat{c}_{\Gamma} z_{h}^{\Gamma} \quad \forall\left(\underline{z}_{h}, \underline{z}_{h}^{\Gamma}\right) \in \underline{P}_{\mathrm{B}, h}^{k} \times \underline{P}_{\Gamma, h}^{k} .
$$

We now prove the discrete counterpart of the Theorem 6.

Theorem 10 (Discrete coercivity). Let $\xi>1 / 2$. Then, for all $\left(\underline{z}_{h}, \underline{z}_{h}^{\Gamma}\right) \in \underline{P}_{\mathrm{B}, h}^{k} \times \underline{P}_{\Gamma, h}^{k}$, it holds

$$
\begin{aligned}
\mathcal{A}_{\xi, h, k}^{\text {transp }}\left(\left(\underline{z}_{h}, \underline{z}_{h}^{\Gamma}\right),\left(\underline{z}_{h}, \underline{z}_{F}^{\Gamma}\right)\right)= & a_{D, h}^{k}\left(\underline{z}_{h}, \underline{z}_{h}\right)+a_{D, h}^{\Gamma, k}\left(\underline{z}_{h}^{\Gamma}, \underline{z}_{h}^{\Gamma}\right) \\
& +\sum_{T \in \mathcal{T}_{h}}\left(\left\|\chi_{\mathrm{B}, T}^{1 / 2} z_{T}\right\|_{T}^{2}+\sum_{F \in \mathcal{F}_{T}} \frac{1}{2}\left\|\left|u_{T F}\right|^{1 / 2}\left(z_{T}-z_{T F}\right)\right\|_{F}^{2}\right) \\
& +\sum_{F \in \mathcal{F}_{h}^{\Gamma}}\left(\left\|\chi_{\Gamma, F}^{1 / 2} z_{F}^{\Gamma}\right\|_{F}^{2}+\sum_{V \in \mathcal{V}_{F}} \frac{1}{2}\left|u_{F V}^{\Gamma}\right|\left(z_{F}^{\Gamma}(V)-z_{V}^{\Gamma}\right)^{2}\right) \\
& +\sum_{F \in \mathcal{F}_{h}^{\Gamma}}\left(\beta_{F}^{\xi}\left\|\left\{\left\{\underline{z}_{h}\right\}\right\}_{F}-z_{F}^{\Gamma}\right\|_{F}^{2}+\beta_{F}\left\|\llbracket \underline{c}_{h} \rrbracket_{F}\right\|_{F}^{2}\right),
\end{aligned}
$$

where, for all $T \in \mathcal{T}_{h}$ and all $F \in \mathcal{F}_{h}^{\Gamma}, \chi_{\mathrm{B}, T}:=\left(\chi_{\mathrm{B}}\right)_{\mid T}$ and $\chi_{\Gamma, F}:=\left(\chi_{\Gamma}\right)_{\mid F}$, respectively.

Remark 11 (Upwind contributions). Unlike the continuous case (see Theorem 6), we have in the second and third lines of the energy balance (41) upwind-related contributions of bulk and fracture region, respectively. These could be removed at the price of having coercivity in a weaker norm.

Proof. The proof is similar to the one of the Theorem 6. Let $\left(\underline{z}_{h}, \underline{z}_{h}^{\Gamma}\right) \in \underline{P}_{\mathrm{B}, h}^{k} \times \underline{P}_{\Gamma, h}^{k}$ be fixed and set $\left(\underline{z}_{h}, \underline{z}_{h}^{\Gamma}\right) \in \underline{P}_{\mathrm{B}, h}^{2 k} \times \underline{P}_{\Gamma, h}^{2 k}$ such that,

$$
\begin{array}{cl}
\forall T \in \mathcal{T}_{h}, \quad\left(\underline{\boldsymbol{z}}_{h}\right)_{\mid T}=\underline{\boldsymbol{z}}_{T}=\left(\boldsymbol{z}_{T},\left(\boldsymbol{z}_{T F}\right)_{F \in \mathcal{F}_{T}}\right):=\left(\frac{z_{T}^{2}}{2},\left(\frac{z_{T F}^{2}}{2}\right)_{F \in \mathcal{F}_{T}}\right), \\
\forall F \in \mathcal{F}_{h}^{\Gamma}, \quad\left(\underline{z}_{h}^{\Gamma}\right)_{\mid F}=\underline{z}_{F}^{\Gamma}=\left(z_{F}^{\Gamma},\left(z_{V}^{\Gamma}\right)_{V \in \mathcal{V}_{F}}\right):=\left(\frac{\left(z_{F}^{\Gamma}\right)^{2}}{2},\left(\frac{\left(z_{V}^{\Gamma}\right)^{2}}{2}\right)_{V \in \mathcal{V}_{F}}\right) .
\end{array}
$$


Using the definition of the global bilinear form $\mathcal{A}_{\xi, h, k}^{\text {transp }}$ (39) with $\left(\underline{c}_{h}, \underline{c}_{h}^{\Gamma}\right)=\left(\underline{z}_{h}, \underline{z}_{h}^{\Gamma}\right)$, we immediately obtain the terms in the first and last line of (41). Let now $\left.\mathcal{I}_{1}:=a_{\boldsymbol{u}, f, h}^{k}\left(\underline{z}_{h}, \underline{z}_{h}\right), \mathcal{I}_{2}:=a_{\boldsymbol{u}, f, h}^{\Gamma, k} \underline{z}_{h}^{\Gamma}, \underline{z}_{h}^{\Gamma}\right)$, and let $\mathcal{I}_{3}$ gather the remaining coupling terms, that is to say, the two last lines on the right-hand side of (39) with $\underline{c}_{h}=\underline{z}_{h}$ and $c_{F}^{\Gamma}=z_{F}^{\Gamma}$ for all $F \in \mathcal{F}_{h}^{\Gamma}$. Expanding $\mathcal{I}_{1}$ and $\mathcal{I}_{2}$ according to their respective definitions (37), and recalling the definitions of the stabilization bilinear forms $s_{\boldsymbol{u}, T}^{k}$ and $s_{\boldsymbol{u}, F}^{k}$ (38), it is inferred that

$$
\begin{aligned}
& \mathcal{I}_{1}=\sum_{T \in \mathcal{T}_{h}}\left(\int_{T}\left(-\boldsymbol{u}_{T} \cdot \nabla \boldsymbol{z}_{T}+f^{-} z_{T}^{2}\right)+\sum_{F \in \mathcal{F}_{T}} \int_{F}\left(u_{T F}\left(\boldsymbol{z}_{T}-\boldsymbol{z}_{T F}\right)+\frac{1}{2}\left|u_{T F}\right|\left(z_{T}-z_{T F}\right)^{2}\right)\right), \\
& \mathcal{I}_{2}=\sum_{F \in \mathcal{F}_{h}^{\Gamma}}\left(\int_{F}\left(-\boldsymbol{u}_{F}^{\Gamma} \cdot \nabla_{\tau} \boldsymbol{z}_{F}^{\Gamma}+\ell_{\Gamma} f_{\Gamma}^{-} z_{F}^{\Gamma^{2}}\right)+\sum_{V \in \mathcal{V}_{F}}\left(u_{F V}^{\Gamma}\left(\boldsymbol{z}_{F}^{\Gamma}(V)-\boldsymbol{z}_{V}^{\Gamma}\right)+\frac{1}{2}\left|u_{F V}^{\Gamma}\right|\left(z_{F}^{\Gamma}(V)-z_{V}^{\Gamma}\right)^{2}\right)\right) .
\end{aligned}
$$

Using the local balances (34) in the bulk and (35) inside the fracture (that hold since $\boldsymbol{z}_{T} \in \mathbb{P}^{2 k}(T)$ for all $T \in \mathcal{T}_{h}$ and $\underline{\underline{z}}_{F}^{\Gamma} \in \underline{P}_{\Gamma, F}^{2 k}$ for all $F \in \mathcal{F}_{h}^{\Gamma}$ ) together with the fact that

$$
\sum_{T \in \mathcal{T}_{h}} \sum_{F \in \mathcal{F}_{T}} u_{T F} \underline{z}_{T F}=\sum_{F \in \mathcal{F}_{h}^{\Gamma}} \llbracket \underline{u}_{h} \underline{\underline{z}}_{h} \rrbracket_{F},
$$

which follows from $\left(\underline{\boldsymbol{u}}_{h}, \underline{\boldsymbol{z}}_{h}\right) \in \underline{\boldsymbol{U}}_{h}^{2 k} \times \underline{P}_{\mathrm{B}, h}^{2 k}$, we finally get from (43a) and (43b)

Referee

\#3,

$$
\begin{aligned}
& \mathcal{I}_{1}=\sum_{T \in \mathcal{T}_{h}}\left(\left\|\chi_{\mathrm{B}, T}^{1 / 2} z_{T}\right\|_{T}^{2}+\sum_{F \in \mathcal{F}_{T}} \frac{1}{2}\left\|\left|u_{T F}\right|^{1 / 2}\left(z_{T}-z_{T F}\right)\right\|_{F}^{2}\right)-\sum_{F \in \mathcal{F}_{h}^{\Gamma}} \int_{F} \llbracket \underline{\boldsymbol{u}}_{h} \underline{\underline{z}}_{h} \rrbracket_{F}, \\
& \mathcal{I}_{2}=\sum_{F \in \mathcal{F}_{h}^{\Gamma}}\left(\left\|\chi_{\Gamma, F}^{1 / 2} z_{F}^{\Gamma}\right\|_{F}^{2}+\sum_{V \in \mathcal{V}_{F}} \frac{1}{2}\left|u_{F V}^{\Gamma}\right|\left(z_{F}^{\Gamma}(V)-z_{V}^{\Gamma}\right)^{2}\right)+\sum_{F \in \mathcal{F}_{h}^{\Gamma}} \int_{F} \llbracket \underline{\boldsymbol{u}}_{h} \rrbracket_{F} \boldsymbol{z}_{F}^{\Gamma} .
\end{aligned}
$$

To conclude, it suffices to prove that the sum of the last term in the right-hand side of (44a) and the last term in the right-hand side of (44b) and $\mathcal{I}_{3}$ is equal to zero. Using (19) to infer first that $\llbracket \underline{\boldsymbol{u}}_{h} \underline{\boldsymbol{z}}_{h} \rrbracket_{F}=\llbracket \underline{\boldsymbol{u}}_{h} \rrbracket_{F}\left\{\left\{\underline{\boldsymbol{z}}_{h}\right\}\right\}_{F}+\left\{\left\{\underline{\boldsymbol{u}}_{h}\right\}\right\}_{F} \llbracket \underline{\boldsymbol{z}}_{h} \rrbracket_{F}$ and then that $\llbracket \underline{\boldsymbol{z}}_{h} \rrbracket_{F}=\llbracket\left[\underline{z}_{h} \rrbracket_{F}\left\{\left\{\underline{z}_{h}\right\}\right\}_{F}\right.$, we get

$$
\sum_{F \in \mathcal{F}_{h}^{\Gamma}} \int_{F}\left(\llbracket \underline{\boldsymbol{u}}_{h} \rrbracket_{F} \underline{\boldsymbol{z}}_{F}^{\Gamma}-\llbracket \underline{\boldsymbol{u}}_{h} \underline{\boldsymbol{z}}_{h} \rrbracket_{F}\right)+\mathcal{I}_{3}=\sum_{F \in \mathcal{F}_{h}^{\Gamma}} \int_{F}\left(\llbracket \underline{\boldsymbol{u}}_{h} \rrbracket_{F}\left(1 / 2\left\{\left\{\underline{z}_{h}\right\}\right\}_{F}^{2}+1 / 8 \llbracket \llbracket_{h} \rrbracket_{F}^{2}-\left\{\left\{\underline{\boldsymbol{z}}_{h}\right\}\right\}_{F}\right)\right),
$$

that concludes the proof since $\left\{\left\{\underline{z}_{h}\right\}\right\}_{F}=\frac{1}{2}\left(\left\{\left\{\underline{z}_{h}\right\}\right\}_{F}^{2}+\frac{1}{4} \llbracket \underline{z}_{h} \rrbracket_{F}^{2}\right)$.

Remark 12 (Polynomial degree and local conservation). The use of polynomials of degree $2 k$ to solve the discrete flow problem (40) is required in the proof of Theorem 10. Indeed, to pass from (43) to (44), the argument is that both the local balances (34) and (35) are valid when we use as test functions $\underline{z}_{h} \in \underline{P}_{\mathrm{B}, h}^{2 k}$ and $\underline{\boldsymbol{z}}_{h}^{\Gamma} \in \underline{P}_{\Gamma, h}^{2 k}$ defined by (42).

Referee

\subsection{Extension to the unsteady case}

In the numerical tests of Sections 5.2-5.3 below, we consider the physically relevant situation of unsteady passive transport with a steady Darcy velocity field. The extension of the HHO scheme (40) to this situation is briefly discussed in what follows.

The transport problem can be extended to the unsteady case by assuming that the unknowns depend on time and adding the unsteady contributions $\phi \mathrm{d}_{t} c$ and $\ell_{\Gamma} \phi_{\Gamma} \mathrm{d}_{t} c_{\Gamma}$ in, respectively, (7a) 
and (8a), where $\phi: \Omega_{\mathrm{B}} \rightarrow \mathbb{R}$ and $\phi_{\Gamma}: \Gamma \rightarrow \mathbb{R}$ stand, respectively, for the porosity in the bulk region and in the fracture such that $0<\phi<1$ and $0<\phi_{\Gamma}<1$. In the numerical tests, we assume that these quantities are piecewise constant on the partitions $\mathcal{P}_{\mathrm{B}}$ and $\mathcal{P}_{\Gamma}$ (see (2) and (3)), respectively. More generally, the porosities could also depend on time. Initial conditions for the bulk and the fracture concentration $c(t=0, \cdot)=c^{0}(\cdot)$ and $c_{\Gamma}(t=0, \cdot)=c_{\Gamma}^{0}(\cdot)$ close the problem. The functions $\widehat{c}$ and $\widehat{c_{\Gamma}}$ that represent the concentration of solute as it is injected in, respectively, the bulk and the fracture, will also be allowed to depend on time.

To discretize in time, we consider for sake of simplicity a uniform partition $\left(t^{n}\right)_{0 \leq n \leq N}$ of the time interval $\left[0, t_{F}\right]$ with $t^{0}=0, t^{N}=t_{F}$ the final time of computation, and $t^{n}-t^{n-1}=\delta t$ the constant time step for all $1 \leq n \leq N$. For any sufficiently regular function of time $\varphi$ taking values in a vector space $V$, we denote by $\varphi^{n} \in V$ its value at discrete time $t^{n}$ and we introduce the backward differencing operator $\delta_{\mathrm{t}}$ such that, for all $1 \leq n \leq N$,

$$
\delta_{\mathrm{t}} \varphi^{n}:=\frac{\varphi^{n}-\varphi^{n-1}}{\delta t} \in V .
$$

With this notation, the discrete problem reads: For all $1 \leq n \leq N$, find $\left(\underline{c}_{h}^{n}, \underline{c}_{h}^{\Gamma, n}\right) \in \underline{P}_{\mathrm{B}, h}^{k} \times \underline{P}_{\Gamma, h}^{k}$ such that, for all $\left(\underline{z}_{h}, \underline{z}_{h}^{\Gamma}\right) \in \underline{P}_{\mathrm{B}, h}^{k} \times \underline{P}_{\Gamma, h}^{k}$,

$$
\int_{\Omega_{\mathrm{B}}} \phi \delta_{\mathrm{t}} c_{h}^{n} z_{h}+\int_{\Gamma} \ell_{\Gamma} \phi_{\Gamma} \delta_{\mathrm{t}} c_{h}^{\Gamma, n} z_{h}^{\Gamma}+\mathcal{A}_{\xi, h, k}^{\mathrm{transp}}\left(\left(\underline{c}_{h}^{n}, \underline{c}_{h}^{\Gamma, n}\right),\left(\underline{z}_{h}, \underline{z}_{F}^{\Gamma}\right)\right)=\int_{\Omega_{\mathrm{B}}} f^{+} \widehat{c}^{n} z_{h}+\int_{\Gamma} \ell_{\Gamma} f_{\Gamma}^{+} \widehat{c}_{\Gamma}^{n} z_{h}^{\Gamma} .
$$

The initial condition is discretized taking $c_{h}^{0}$ and $c_{h}^{\Gamma, 0}$ equal to the $L^{2}$-orthogonal projections on $\mathbb{P}^{k}\left(\mathcal{T}_{h}\right)$ and $\mathbb{P}^{l}\left(\mathcal{F}_{h}^{\Gamma}\right)$ of $c^{0}$ and $c_{\Gamma}^{0}$, respectively. Notice that it is not necessary to prescribe face values for the concentration in the bulk region, nor vertex values for the concentration in the fracture, as these discrete unknowns do not appear in the discretization of the time derivative.

\section{Numerical results}

This section contains an extensive numerical validation of the HHO method. We first study numerically the convergence rates achieved by the method, and then propose two more physical test cases in which fractures act as barriers or conduits, depending on the value of the permeability parameters.

\subsection{Convergence for a steady problem}

We start by a numerical study of the convergence rates of the method for both the flow problem (31) and the steady passive transport problem (40).

Referee \#1, typos

\subsubsection{Analytical solution}

We approximate problems (31) and (40) on the square domain $\Omega=(0,1)^{2}$ crossed by the fracture $\Gamma=\left\{\boldsymbol{x} \in \Omega: x_{1}=0.5\right\}$, and set $\ell_{\Gamma}=0.01$ and $\xi=3 / 4$. For the flow problem, we consider the exact solutions corresponding to the bulk and fracture pressures

$$
p(\boldsymbol{x}):=\left\{\begin{array}{ll}
\cos \left(2 x_{1}\right) \cos \left(\pi x_{2}\right) & \text { if } x_{1}<0.5 \\
\cos \left(\pi x_{1}\right) \cos \left(\pi x_{2}\right) & \text { if } x_{1}>0.5
\end{array}, \quad p_{\Gamma}(\boldsymbol{x}):=\{\{c\}\}_{\Gamma}-\lambda_{\Gamma} \llbracket \boldsymbol{u} \rrbracket_{\Gamma} \cdot \boldsymbol{n}_{\Gamma},\right.
$$

and let $\boldsymbol{u}_{\mid \Omega_{\mathrm{B}, i}}=-\boldsymbol{K} \nabla p_{\mid \Omega_{\mathrm{B}, i}}$ for $i \in\{1,2\}$ and $\boldsymbol{u}_{\Gamma}=-K_{\Gamma} \nabla_{\tau} p_{\Gamma}$, with $\kappa_{\Gamma}^{\tau}=1, \kappa_{\Gamma}^{n}=0.01$ and

$$
\boldsymbol{K}:=\frac{\cos (1)}{\sin (1)+\pi / 2}\left[\begin{array}{cc}
\kappa_{\Gamma}^{n} /\left(2 \ell_{\Gamma}\right) & 0 \\
0 & 1
\end{array}\right], \quad K_{\Gamma}:=\kappa_{\Gamma}^{\tau} \ell_{\Gamma} .
$$


For the steady passive transport problem, the exact solutions corresponding to the bulk and fracture concentrations are given by

$$
\begin{aligned}
c(\boldsymbol{x}) & :=\left\{\begin{array}{ll}
\exp \left(2 / \pi \cos \left(\pi x_{1}\right)\left(\beta_{\Gamma}-\frac{1}{8} \cos \left(\pi x_{2}\right) \frac{\kappa_{\Gamma}^{n}}{\ell_{\Gamma}} \frac{\cos (1)(\sin (1)-\pi / 2)}{\sin (1)+\pi / 2}\right)\right) & \text { if } x_{1}<0.5 \\
\exp \left(2 / \pi\left(\cos \left(\pi x_{1}\right)-\pi\right)\left(\beta_{\Gamma}-\frac{1}{8} \cos \left(\pi x_{2}\right) \frac{\left.\left.\kappa_{\Gamma}^{n} \frac{\cos (1)(\sin (1)-\pi / 2)}{\ell_{\Gamma}}\right)\right)}{\sin (1)+\pi / 2}\right)\right. & \text { if } x_{1}>0.5
\end{array},\right. \\
c_{\Gamma}(\boldsymbol{x}): & =\frac{\llbracket \boldsymbol{u} c-\boldsymbol{D} \nabla c \rrbracket_{\Gamma} \cdot \boldsymbol{n}_{\Gamma}-\{\{c\}\}_{\Gamma}\left(1 / 2 \llbracket \boldsymbol{u} \rrbracket_{\Gamma} \cdot \boldsymbol{n}_{\Gamma}+\beta_{\Gamma}^{\xi}\right)}{1 / 2 \llbracket \boldsymbol{u} \rrbracket_{\Gamma} \cdot \boldsymbol{n}_{\Gamma}-\beta_{\Gamma}^{\xi}},
\end{aligned}
$$

Referee \#3,

comment

with $\boldsymbol{D}=\boldsymbol{I}_{2}$, the identity matrix of $\mathbb{R}^{2 \times 2}, \mathcal{D}_{\Gamma}^{\tau}=1$ and $\mathcal{D}_{\Gamma}^{n}=0.01$. The source terms $f, f_{\Gamma}$ are inferred from (1b) and (1c), respectively. The right-hand sides of (7a) and (8a) are also modified by introducing nonzero terms in accordance with the expressions of $c$ and $c_{\Gamma}$; see (46). It can be checked that, with this choice of analytical solutions, the jump and average of $p, \boldsymbol{u}, c, \boldsymbol{D} \nabla c$ are not identically zero on the fracture, which enables us to test the weak enforcement of the transmission conditions (4) for the flow problem and (9) for the steady passive transport problem.

\subsubsection{Error measures}

On the spaces of discrete bulk unknowns $\underline{\boldsymbol{U}}_{h}^{2 k}$ and $\underline{P}_{h}^{k}$, we define the norms $\|\cdot\|_{\boldsymbol{U}, h}$ and $\|\cdot\|_{D, h}$ such that, for all $\underline{\boldsymbol{q}}_{h} \in \underline{\boldsymbol{U}}_{h}^{2 k}$ and all $\underline{z}_{h} \in \underline{P}_{h}^{k}$,

$$
\begin{aligned}
\left\|\underline{\boldsymbol{q}}_{h}\right\|_{\boldsymbol{U}, h}^{2} & :=\sum_{T \in \mathcal{T}_{h}}\left(\bar{K}_{\mathrm{B}, T}\right)^{-1}\left(\left\|\boldsymbol{q}_{T}\right\|_{T}^{2}+\sum_{F \in \mathcal{F}_{T}} h_{F}\left\|q_{T F}\right\|_{F}^{2}\right), \\
\left\|\underline{z}_{h}\right\|_{D, h}^{2}: & :=\sum_{T \in \mathcal{T}_{h}} \varrho_{\boldsymbol{D}, T}^{-1}\left(\left\|\boldsymbol{D}_{T}^{1 / 2} \nabla z_{T}\right\|_{T}^{2}+\sum_{F \in \mathcal{F}_{T}} \frac{D_{T F}}{h_{F}}\left\|z_{T}-z_{T F}\right\|_{F}^{2}\right),
\end{aligned}
$$

where, for any $T \in \mathcal{T}_{h}, \bar{K}_{\mathrm{B}, T}$ is the largest eigenvalue of the (constant) permeability tensor $\boldsymbol{K}_{T}$, while $\varrho_{\boldsymbol{D}, T}:=\bar{D}_{\mathrm{B}, T} / \underline{D}_{\mathrm{B}, T}$ is the bulk anisotropy ratio with $\bar{D}_{\mathrm{B}, T}, \underline{D}_{\mathrm{B}, T}>0$ denoting, respectively, the largest and smallest eigenvalue of the (constant) local bulk diffusion-dispersion tensor $\boldsymbol{D}_{T}$.

On the spaces of discrete fracture unknowns $\underline{P}_{\Gamma, h}^{2 k}$ and $\underline{P}_{\Gamma, h}^{k}$ we define the norms $\|\cdot\|_{\Gamma, K, h}$ and $\|\cdot\|_{\Gamma, D, h}$ such that, for all $\underline{v}_{h}^{\Gamma} \in \underline{P}_{\Gamma, h}^{2 k}$ and all $\underline{z}_{h}^{\Gamma} \in \underline{P}_{\Gamma, h}^{k}$,

$$
\begin{aligned}
\left\|\underline{v}_{h}^{\Gamma}\right\|_{\Gamma, K, h}^{2}:=\sum_{F \in \mathcal{F}_{h}^{\Gamma}}\left(\left\|K_{F}^{1 / 2} \nabla_{\tau} v_{F}^{\Gamma}\right\|_{F}^{2}+\sum_{V \in \mathcal{V}_{F}} \frac{K_{F}}{h_{F}}\left(v_{F}^{\Gamma}(V)-v_{V}^{\Gamma}\right)^{2}\right), \\
\left\|\underline{z}_{h}^{\Gamma}\right\|_{\Gamma, D, h}^{2}:=\sum_{F \in \mathcal{F}_{h}^{\Gamma}}\left(\left\|D_{F}^{1 / 2} \nabla_{\tau} z_{F}^{\Gamma}\right\|_{F}^{2}+\sum_{V \in \mathcal{V}_{F}} \frac{D_{F}}{h_{F}}\left(z_{F}^{\Gamma}(V)-z_{V}^{\Gamma}\right)^{2}\right) .
\end{aligned}
$$

For the flow problem, we monitor the following errors defined as the difference between the numerical solution and suitable projections of the exact solution:

$$
\left\|\underline{\boldsymbol{u}}_{h}-\underline{\boldsymbol{I}}_{h}^{2 k} \boldsymbol{u}\right\|_{\boldsymbol{U}, h}, \quad\left\|\underline{p}_{h}^{\Gamma}-\underline{I}_{h}^{2 k} p_{\Gamma}\right\|_{\Gamma, K, h}, \quad\left\|p_{h}-\pi_{h}^{2 k} p\right\|_{L^{2}\left(\Omega_{\mathrm{B}}\right)}, \quad\left\|p_{h}^{\Gamma}-\pi_{\Gamma, h}^{2 k} p_{\Gamma}\right\|_{L^{2}(\Gamma)},
$$

where $\underline{\boldsymbol{I}}_{h}^{2 k} \boldsymbol{u}:=\left(\boldsymbol{K}_{T} \nabla y_{T},\left(\pi_{F}^{2 k}\left(\boldsymbol{u} \cdot \boldsymbol{n}_{T F}\right)_{F \in \mathcal{F}_{T}}\right)_{T \in \mathcal{T}_{h}}\right.$ with $y_{T} \in \mathbb{P}^{2 k}(T)$ is such that $\int_{T}\left(\boldsymbol{K}_{T} \nabla y_{T}-\boldsymbol{u}\right)$. $\nabla v_{T}=0$ for all $v_{T} \in \mathbb{P}^{2 k}(T), \underline{I}_{\Gamma, h}^{2 k} p_{\Gamma}:=\left(\left(\pi_{F}^{2 k} p_{\Gamma \mid F}\right)_{F \in \mathcal{F}_{h}^{\Gamma}},\left(p_{\Gamma}(V)\right)_{V \in \mathcal{V}_{h}}\right)$ with $\pi_{F}^{2 k}$ denoting the $L^{2}-$ orthogonal projector on $\mathbb{P}^{2 k}(F)$, and $\pi_{h}^{2 k} p$ and $\pi_{\Gamma, h}^{2 k} p_{\Gamma}$ denote, respectively, the $L^{2}$-orthogonal projections of $p$ and $p_{\Gamma}$ on $P_{\mathrm{B}, h}^{2 k}$ and $P_{\Gamma, h}^{2 k}$, 


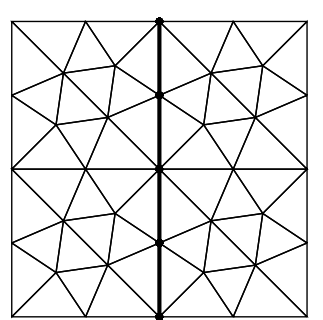

(a) Triangular

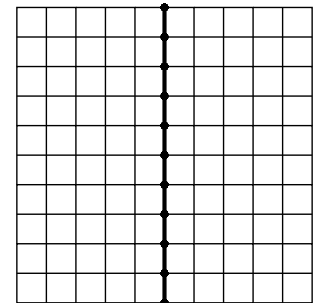

(b) Cartesian

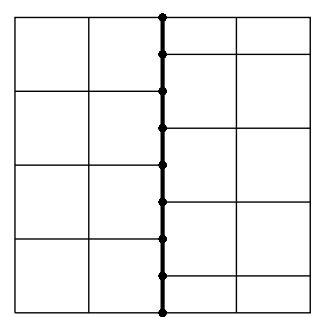

(c) Nonconforming

Figure 3: Mesh families for the numerical tests

Similarly, for the steady passive transport problem we consider the following error measures:

$$
\left\|\underline{c}_{h}-\underline{I}_{h}^{k} c\right\|_{D, h}, \quad\left\|\underline{c}_{h}^{\Gamma}-\underline{I}_{\Gamma, h}^{k} c_{\Gamma}\right\|_{\Gamma, D, h}, \quad\left\|c_{h}-\pi_{h}^{k} c\right\|_{L^{2}\left(\Omega_{\mathrm{B}}\right)}, \quad\left\|c_{h}^{\Gamma}-\pi_{\Gamma, h}^{k} c_{\Gamma}\right\|_{L^{2}(\Gamma)},
$$

where $\underline{I}_{h}^{k} c:=\left(\left(\pi_{T}^{k} c_{\mid T}\right)_{T \in \mathcal{T}_{h}},\left(\pi_{F}^{k}\left(c_{\mid F}\right)\right)_{F \in \mathcal{F}_{h}}\right)$ with $\pi_{T}^{k}$ and $\pi_{F}^{k}$ denoting, respectively, the $L^{2}$-orthogonal projectors on $\mathbb{P}^{k}(T)$ and $\mathbb{P}^{k}(F), \underline{I}_{\Gamma, h}^{k} c_{\Gamma}:=\left(\left(\pi_{F}^{k} c_{\Gamma \mid F}\right)_{F \in \mathcal{F}_{h}^{F}},\left(c_{\Gamma}(V)\right)_{V \in \mathcal{V}_{h}}\right)$, and $\pi_{h}^{k} c$ and $\pi_{\Gamma, h}^{k} c_{\Gamma}$ denote, respectively, the $L^{2}$-orthogonal projections of $c$ and $c_{\Gamma}$ on $P_{\mathrm{B}, h}^{k}$ and $P_{\Gamma, h}^{k}$.

\subsubsection{Results}

We consider the triangular, Cartesian and nonconforming mesh families of Figure 3.

In Figure 4, we display the errors (47) for the flow problem as functions of the meshsize. The flow problem (31) is solved using polynomials two times higher than for the passive transport problem, so higher convergence rates than for the passive transport problem are to be expected. More specifically, on the triangular mesh we observe convergence in $h^{2 k+1}$ of the discretization error measured in the energy-like norms $\left\|\underline{\boldsymbol{u}}_{h}-\underline{\boldsymbol{I}}_{h}^{2 k} \boldsymbol{u}\right\|_{\boldsymbol{P}, h}$ and $\left\|\underline{p}_{h}^{\Gamma}-\underline{\boldsymbol{I}}_{h}^{2 k} p_{\Gamma}\right\|_{\Gamma, K, h}$, and convergence in $h^{2 k+2}$ for the error measured in the $L^{2}$-norms $\left\|p_{h}-\pi_{h}^{2 k} p\right\|_{L^{2}\left(\Omega_{\mathrm{B}}\right)}$ and $\left\|p_{h}^{\Gamma}-\pi_{\Gamma, h}^{2 k} p_{\Gamma}\right\|_{L^{2}(\Gamma)}$. Slightly better convergence rates are observed on Cartesian and nonconforming meshes, as already noticed in [15].

For the steady passive transport problem (40), we plot in Figure 5 the errors (48) as functions of the meshsize. For both the energy-like norms of the error $\left\|\underline{c}_{h}-\underline{I}_{h}^{k} c\right\|_{D, h}$ and $\left\|\underline{c}_{h}^{\Gamma}-\underline{I}_{\Gamma, h}^{k} c_{\Gamma}\right\|_{\Gamma, D, h}$, we obain convergence in $h^{k+1}$. For the $L^{2}$-norms of the error $\left\|c_{h}-\pi_{h}^{k} c\right\|_{L^{2}\left(\Omega_{\mathrm{B}}\right)}$ and $\left\|c_{h}^{\Gamma}-\pi_{\Gamma, h}^{k} c\right\|_{L^{2}(\Gamma)}$, on the other hand, we obtain convergence in $h^{k+2}$ using piecewise linear or quadratic polynomials, and for the case $k=0$ in a fracture, we remark a stagnation of convergence around $10^{-3}$. This phenomenon will be investigate in further works.

\subsection{Unsteady transport with impermeable fractures}

We next consider a physical test case modelling the unsteady passive displacement of a solute in a porous medium in which the fractures act as barriers.

The configuration is depicted in Figure 6a. More specifically, the computational domain is the unit square $\Omega=(0,1)^{2}$, with fractures of constant thickness $\ell_{\Gamma}=10^{-2}$ corresponding to

$$
\Gamma=\left\{\boldsymbol{x}=\left(x_{1}, x_{2}\right) \in \Omega:\left(x_{1}<0.75 \text { and } x_{2} \in\{0.25,0.75\}\right) \text { or }\left(x_{1}>0.25 \text { and } x_{2}=0.5\right)\right\} .
$$

The injection well is located in $(0.5,0)$, the production one in $(0.5,1)$, and both are modeled by the source term $f$ defined such that

$$
f(\boldsymbol{x})=\frac{1}{2}\left(\tanh \left(200\left(0.025-\sqrt{\left(x_{1}^{2}-0.5\right)+x_{2}^{2}}\right)\right)-\tanh \left(200\left(0.025-\sqrt{\left(x_{1}^{2}-0.5\right)+\left(x_{2}-1\right)^{2}}\right)\right)\right) .
$$

Referee \#3, comment 4

Referee \#3, comment 5 
Referee
The fracture source term $f_{\Gamma}$ is set to 0 . It can be checked that the average of $f$ in $\Omega_{\mathrm{B}}$ is zero, so the compatibility condition (5) is verified. We set the user parameter $\xi=0.75$.

Concerning the flow problem, we select the values of the permeability in the bulk and in the fracture so as to obtain impermeable fractures. More specifically, in the bulk we set $\boldsymbol{K}=10^{-3} \boldsymbol{I}$, while in the fractures the tangential and normal permeability are, respectively, $\kappa_{\Gamma}^{\tau}=10^{-3}$ and $\kappa_{\Gamma}^{n}=10^{-6}$. In Figure 6a, we display the bulk pressure $p$ obtained with such parameters and the plot over $x_{1}=0.5$. We can clearly see that the pressure jumps across the fractures and decreases from the injection to the production well.

We consider the unsteady passive transport problem (45), set the final time $t_{F}=100$ and the time step $\delta t=1$. At $t=0$, there is not solute in the bulk nor in the fractures. The concentration of injected solute in the bulk is given, for all $x \in \Omega_{\mathrm{B}}$, by $\widehat{c}(t, \boldsymbol{x})=1$ if $t<30$ and $\widehat{c}(t, \boldsymbol{x})=0$ otherwise. Since we do not have wells in the fracture, we set $\widehat{c_{\Gamma}} \equiv 0$. The porosity in the bulk and in the fracture is set to $\phi=\phi_{\Gamma}=10^{-1}$. Following $[3,30]$, the diffusion-dispersion tensor in the bulk is defined locally for all $T \in \mathcal{T}_{h}$ such that

$$
\boldsymbol{D}_{T}=\phi d_{\mathrm{m}} \boldsymbol{I}_{2}+\phi\left|\boldsymbol{F}_{T}^{2 k+1} \underline{\boldsymbol{u}}_{T}\right|\left(d_{1} \boldsymbol{E}_{\boldsymbol{u}, T}+d_{\mathrm{t}}\left(\boldsymbol{I}_{2}-\boldsymbol{E}_{\boldsymbol{u}, T}\right)\right),
$$

where $\left|\boldsymbol{F}_{T}^{2 k+1} \underline{\boldsymbol{u}}_{T}\right|$ is the Euclidean norm of $\boldsymbol{F}_{T}^{2 k+1} \underline{\boldsymbol{u}}_{T}, \boldsymbol{E}_{\boldsymbol{u}, T}:=\left|\boldsymbol{F}_{T}^{2 k+1} \underline{\boldsymbol{u}}_{T}\right|^{-2}\left(\boldsymbol{F}_{T}^{2 k+1} \underline{\boldsymbol{u}}_{T} \otimes \boldsymbol{F}_{T}^{2 k+1} \underline{\boldsymbol{u}}_{T}\right)$, while $d_{\mathrm{m}}=10^{-5}, d_{1}=1$ and $d_{\mathrm{t}}=10^{-2}$ denote, respectively, the molecular diffusion, longitudinal, and transverse dispersion coefficients. Notice that the high-order reconstruction of the Darcy velocity is needed to define $\boldsymbol{D}_{T}$ since, if using constant elements $k=0$, we do not have cell-based DOFs for the flux. The fracture counterpart of the diffusion-dispersion coefficient is defined, for all $F \in \mathcal{F}_{h}^{\Gamma}$, as follows

$$
D_{F}=\ell_{\Gamma} \phi_{\Gamma} d_{\mathrm{m}}^{\Gamma} \boldsymbol{I}_{2}+\phi_{\Gamma}\left|\boldsymbol{u}_{F}^{\Gamma}\right|\left(d_{1}^{\Gamma} \boldsymbol{E}_{\boldsymbol{u}, F}+d_{\mathrm{t}}^{\Gamma}\left(\boldsymbol{I}_{2}-\boldsymbol{E}_{\boldsymbol{u}, F}\right)\right),
$$

with $\boldsymbol{E}_{\boldsymbol{u}, F}:=\left|\boldsymbol{u}_{F}^{\Gamma}\right|^{-2}\left(\boldsymbol{u}_{F}^{\Gamma} \otimes \boldsymbol{u}_{F}^{\Gamma}\right)$ and where $d_{\mathrm{m}}^{\Gamma}=10^{-5}, d_{1}^{\Gamma}=1$ and $d_{\mathrm{t}}^{\Gamma}=10^{-2}$ denote, respectively, the fracture molecular diffusion, longitudinal, and transverse dispersion coefficients. We set the normal diffusion-dispersion coefficient of the fracture $\mathcal{D}_{\Gamma}^{n}$ equal to 1 . A more in-depth investigation of the meaning of this term is postponed to a future work.

We run the test case on the Cartesian mesh depicted in Figure $3 \mathrm{~b}$ of meshsize $h=7.81 \cdot 10^{-3}$ with $k=2$. In Figure $6 \mathrm{~b}$, we display the bulk concentration at different time $t$. As expected, the solute follows the corridors designed by the fractures that act as barriers and goes from the injection to the production well.

\subsection{Unsteady transport with permeable fractures}

We next focus on the case where the fractures act as conduits. The domain is still the square unit $\Omega=(0,1)^{2}$, the fractures of constant thickness $\ell_{\Gamma}=10^{-2}$ are located in

$$
\Gamma=\left\{\boldsymbol{x} \in \Omega: x_{1} \in\{2 / 32,8 / 32,13 / 32,19 / 32,24 / 32,30 / 32\} \text { and } 0.25<x_{2}<0.75\right\} .
$$

The configuration is depicted in Figure 7a. The only parameters that differ from the previous test case of Section 5.2 are the fracture permeabilities: to obtain permeable fractures, we set the normal permeability $\kappa_{\Gamma}^{n}=10^{-3}$ and the tangential one $\kappa_{\Gamma}^{\tau}=10^{-1}$. With this choice, it is expected that the flow is attracted by the fractures.

In Figure 7a, we display the bulk pressure $p$ and Darcy velocity $\boldsymbol{u}$ where, for the latter, the color scale correspond to the value of the magnitude. As expected, the flow is from the injection well towards the fractures near the bottom of the domain, and from the fractures to the production well near the top of the domain.
Referee 
Referee In Figure $7 \mathrm{~b}$, we display the bulk concentration $c$ at different times. We can distinctly see that

$\# 2$, the solute channeled by the fractures flows towards the production well faster than the solute in the

typos surrounding bulk medium.

Referee

$\# 1$,

typos

\section{Conclusions}

We conclude this paper by pointing out its main contributions and discuss perspectives of further works. We have introduced a new reduced model for the passive transport of solute in fractured porous media driven by Darcy velocites. To derive transmission conditions, we used an energy-based argument such that, as in the unreduced model, transport terms do not contribute to the energy balance. These transmission conditions allow the solute concentration to jump across the fracture. The presentation of the model and its discretization are done considering the steady case, while the extension to the unsteady case is presented and used for numerical experiments. In future work, we will investigate the meaning of the fracture normal diffusion-dispersion coefficient $\mathcal{D}_{\Gamma}^{n}$ in the new transmission conditions (9) of the new reduced model for passive transport in fractured porous media, and we will carry out the complete analysis of the discrete formulation, including its well-posedness and the study of the convergence properties of the HHO method.

\section{References}

[1] J. Aghili, S. Boyaval, and D. A. Di Pietro. "Hybridization of mixed high-order methods on general meshes and application to the Stokes equations". In: Comput. Meth. Appl. Math. 15.2 (2015), pp. 111-134. DOI: 10.1515/cmam-2015-0004.

[2] C. Alboin, J. Jaffré, J. E. Roberts, and C. Serres. "Modeling fractures as interfaces for flow and transport in porous media". In: Fluid Flow and Transport in Porous Media: Mathematical and Numerical Treatment. Ed. by Amer. Math. Soc. Contemp. Math. Vol. 295. 2002, pp. 13-24. URL: https://who.rocq.inria.fr/Jean. Roberts/2002alboinjaffrerobertsserresAMS-web.pdf.

[3] D. Anderson and J. Droniou. "An arbitrary order scheme on generic meshes for miscible displacements in porous media". In: SIAM J. Sci. Comput. (2018). Accepted for publication. URL: https: //arxiv.org/abs/1707.04038.

[4] P. Angot, F. Boyer, and F. Hubert. "Asymptotic and Numerical Modelling of Flows in Fractured Porous Media". In: ESAIM: Math. Model Numer. Anal. 43.2 (2009), pp. 239-275. Dor: $10.1051 / \mathrm{m} 2 \mathrm{an} / 2008052$.

[5] P. Angot, T. Gallouët, and R. Herbin. "Convergence of finite volume methods on general meshes for non smooth solution of elliptic problems with cracks". In: Finite Volumes for Complex Applications II (1999), pp. 215-222.

[6] P. F. Antonietti, C. Facciola, A. Russo, and M. Verani. Discontinuous Galerkin approximation of flows in fractured porous media on polytopic grids. MOX report No. 55/2016. 2016. URL: https://www . mate . polimi .it/biblioteca/add/qmox/55-2016.pdf.

[7] P. F. Antonietti, L. Formaggia, A. Scotti, M. Verani, and N. Verzotti. "Mimetic finite difference approximation of flows in fractured porous media". In: ESAIM: Math. Model Numer. Anal. 50.3 (2016), pp. 809-832. DOI: $10.1051 / \mathrm{m} 2 \mathrm{an} / 2015087$.

[8] P. Bastian, Z. Chen, R. E. Ewing, R. Helmig, H Jakobs, and V. Reichenberger. "Numerical Simulation of Multiphase Flow in Fractured Porous Media". In: Numerical Treatment of Multiphase Flows in Porous Media 52 (1999), pp. 50-68. Dor: 10 .1007/3-540-45467-5_4. 
[9] M. F. Benedetto, S. Berrone, S. Pieraccini, and S. Scialò. "The Virtual Element Method for discrete fracture network simulations". In: Comput. Meth. Appl. Mech. Engrg. 280 (2014), pp. 135-156. DOI: 10.1016/j . cma.2014.07.016.

[10] S. Berrone, S. Pieraccini, and S. Scialò. "Flow simulations in porous media with immersed intersecting fractures". In: J. Comput. Phys. 345 (2017), pp. 768-791. Dor: 10 . 1016/j . jcp . 2017 . 05 . 049.

[11] S. Berrone, S. Pieraccini, and S. Scialò. "Non-stationary transport phenomena in networks of fractures: Effective simulations and stochastic analysis". In: Comput. Meth. Appl. Mech. Engrg. 315 (2016), pp. 1098-1112. Dor: 10.1016/j . cma.2016.12.006.

[12] D. Boffi and D. A. Di Pietro. "Unified formulation and analysis of mixed and primal discontinuous skeletal methods on polytopal meshes". In: ESAIM: Math. Model Numer. Anal. 52.1 (2018), pp. 1-28. Dor: 10.1051/m2an/2017036.

[13] W. M. Boon and J. M. Nordbotten. Robust discretization of flow in fractured porous media. Submitted. Preprint arXiv:1601.06977 [math.NA]. 2016.

[14] K. Brenner, J. Hennicker, R. Masson, and P Samier. "Hybrid-dimensional modelling of twophase flow through fractured porous media with enhanced matrix fracture transmission conditions". In: Journal of Computational Physics 357 (2018), pp. 100-124. DOI: 10 . 1016/ j . j cp . 2017 . 12 . 003.

[15] F. Chave, D. A. Di Pietro, and L. Formaggia. "A Hybrid High-Order method for Darcy flows in fractured porous media”. In: SIAM J. Sci. Comput. 40.2 (2018), A1063-A1094. DoI: 10.1137/17M1119500.

[16] A. Chernyshenko, M. Olshanskii, and Y. Vassilevski. "A Hybrid Finite Volume-Finite Element Method for bulk-surface coupled problems". In: Journal of Computational Physics 352 (2016), pp. 516-533. Dor: 10.1016/j . jcp. 2017.09.064.

[17] C. D'Angelo and A. Scotti. "A Mixed Finite Element Method for Darcy Flow in Fractured Porous Media with non-matching Grids". In: ESAIM: Math. Model Numer. Anal. 46.2 (2012), pp. 465-489. DOI: $10.1051 / \mathrm{m} 2 \mathrm{an} / 2011148$.

[18] M. Del Pra, A. Fumagalli, and A. Scotti. "Well-posedness of fully coupled fracture/bulk Darcy flow with XFEM". In: SIAM J. Numer. Anal. 55.2 (2017), pp. 785-811. Dor: 10 . 1137/15M1022574.

[19] D. A. Di Pietro and J. Droniou. "A Hybrid High-Order method for Leray-Lions elliptic equations on general meshes". In: Math. Comp. 86.307 (2017), pp. 2159-2191.

[20] D. A. Di Pietro, J. Droniou, and A. Ern. "A discontinuous-skeletal method for advectiondiffusion-reaction on general meshes". In: SIAM J. Numer. Anal. 53.5 (2015), pp. 2135-2157. DOI: $10.1137 / 140993971$.

[21] D. A. Di Pietro, A. Ern, and S. Lemaire. "An arbitrary-order and compact-stencil discretization of diffusion on general meshes based on local reconstruction operators". In: Comput. Meth. Appl. Math. 14.4 (2014). Open access, pp. 461-472. DoI: 10.1515/cmam-2014-0018.

[22] D. A. Di Pietro and R. Tittarelli. "Lectures from the fall 2016 thematic quarter at Institut Henri Poincaré". In: ed. by L. Formaggia D. A. Di Pietro A. Ern. SEMA-SIMAI. Accepted for publication. Springer, 2017. Chap. An introduction to Hybrid High-Order methods. URL: http://arxiv.org/abs/1703.05136.

[23] I. Faille, E. Flauraud, F. Nataf, S. Pégaz-Fiornet, F. Schneider, and F. Willien. "A new fault model in geological basin modelling. Application of finite volume scheme and domain decomposition methods". In: Finite Volumes for Complex Applications III (2002), pp. 543-550. 
[24] B. Flemisch, A. Fumagalli, and Scotti A. "Advances in Discretization Methods". In: ed. by G. Ventura and E. Benvenuti. Vol. 12. SEMA-SIMAI. Springer, 2016. Chap. A Review of the XFEM-Based Approximation of Flow in Fractured Porous Media. Dor: 10. 1007/978-3-319-41246-7_3.

[25] L. Formaggia, A. Quarteroni, and C. Vergara. "On the physical consistency between threedimensional and one-dimensional models in haemodynamics". In: Journal of Computational Physics 244 (2013), pp. 97-112. DOI: 10.1016/j . jcp.2012.08.001.

[26] A. Fumagalli and E. Keilegavlen. "Dual Virtual Element Method for Discrete Fractures Networks”. In: SIAM J. Sci. Comput. 40.1 (2018), B228-B258. Dor: 10. 1137/16M1098231.

[27] A. Fumagalli and A. Scotti. "A reduced model for flow and transport in fractured porous media with non-matching grids". In: Numerical Mathematics and Advanced Applications 2011. 2013, pp. 499-507. DoI: 10.1007/978-3-642-33134-3_53.

[28] S. Gross, M. A. Olshanskii, and A. Reusken. "A trace finite element method for a class of coupled bulk-interface transport problems". In: ESAIM: Math. Model Numer. Anal. 49.5 (2015), pp. 1303-1330. DOI: 10.1051/m2an/2015013.

[29] V. Martin, J. Jaffré, and J. E. Roberts. "Modeling fractures and barriers as interfaces for flow in porous media". In: SIAM J. Matrix Analysis and Applications 26.5 (2005), pp. 1667-1691. DOI: $10.1137 / \mathrm{S} 1064827503429363$.

[30] D. W. Peaceman. "Improved treatment of dispersion in numerical calculation of multidimensional miscible displacement". In: Soc. Petrol. Eng. J. 6 (1966), pp. 213-216. Dor: 10.2118/1362-PA.

[31] A. Scotti, L. Formaggia, and F. Sottocasa. Analysis of a mimetic finite difference approximation offlows in fractured porous media. Accepted for publication. 2017. DoI: 10. 1051/m2an/2017028. 

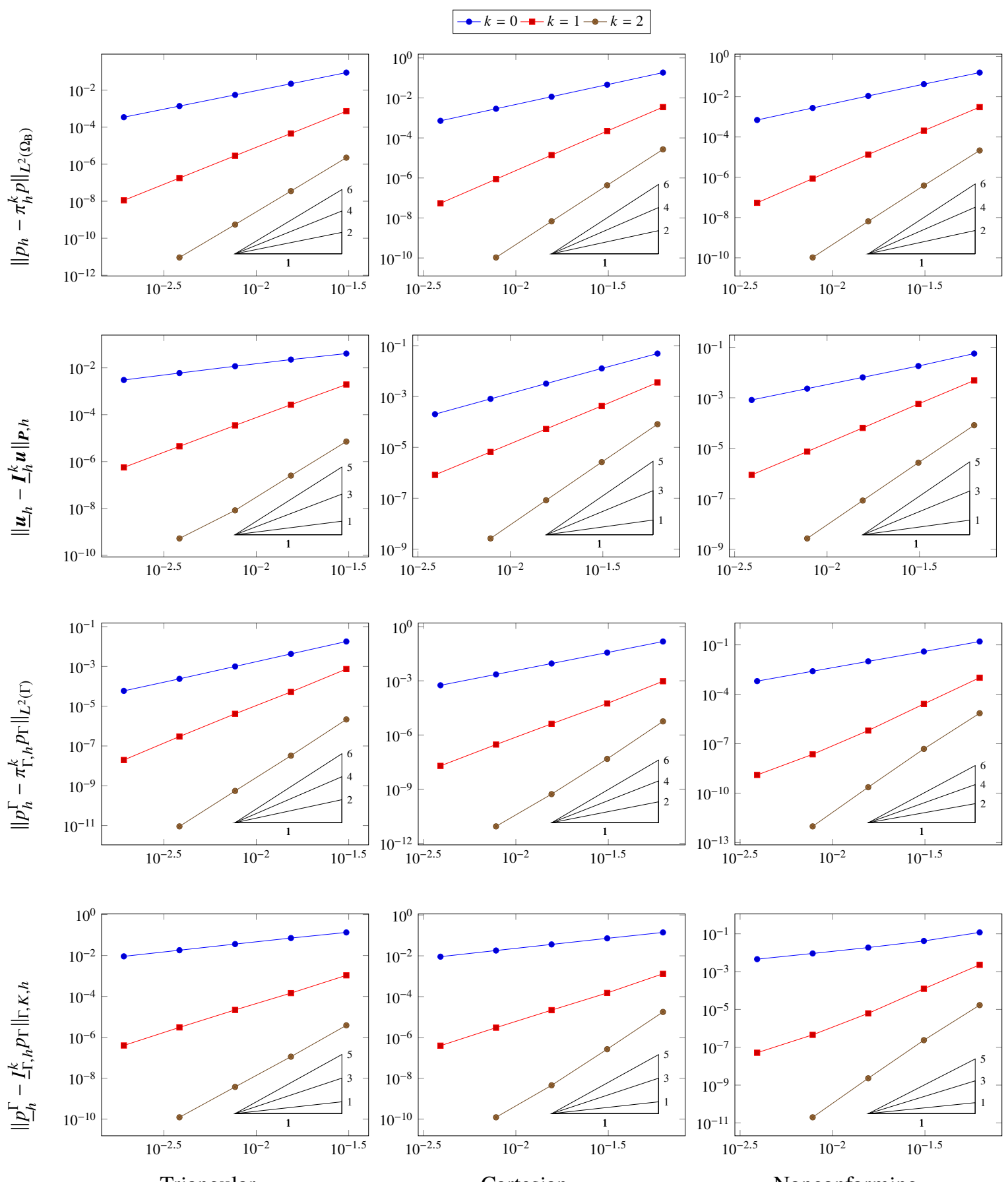

Cartesian

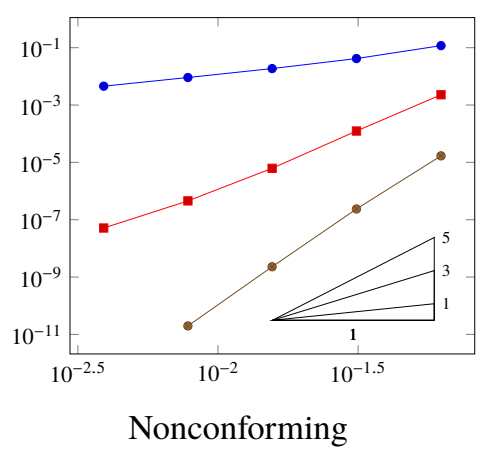

Figure 4: Convergence results for the test case of Section 5.1. Errors (47) for the flow problem v. $h$ on the triangular, Cartesian and nonconforming mesh families of Figure 3. 

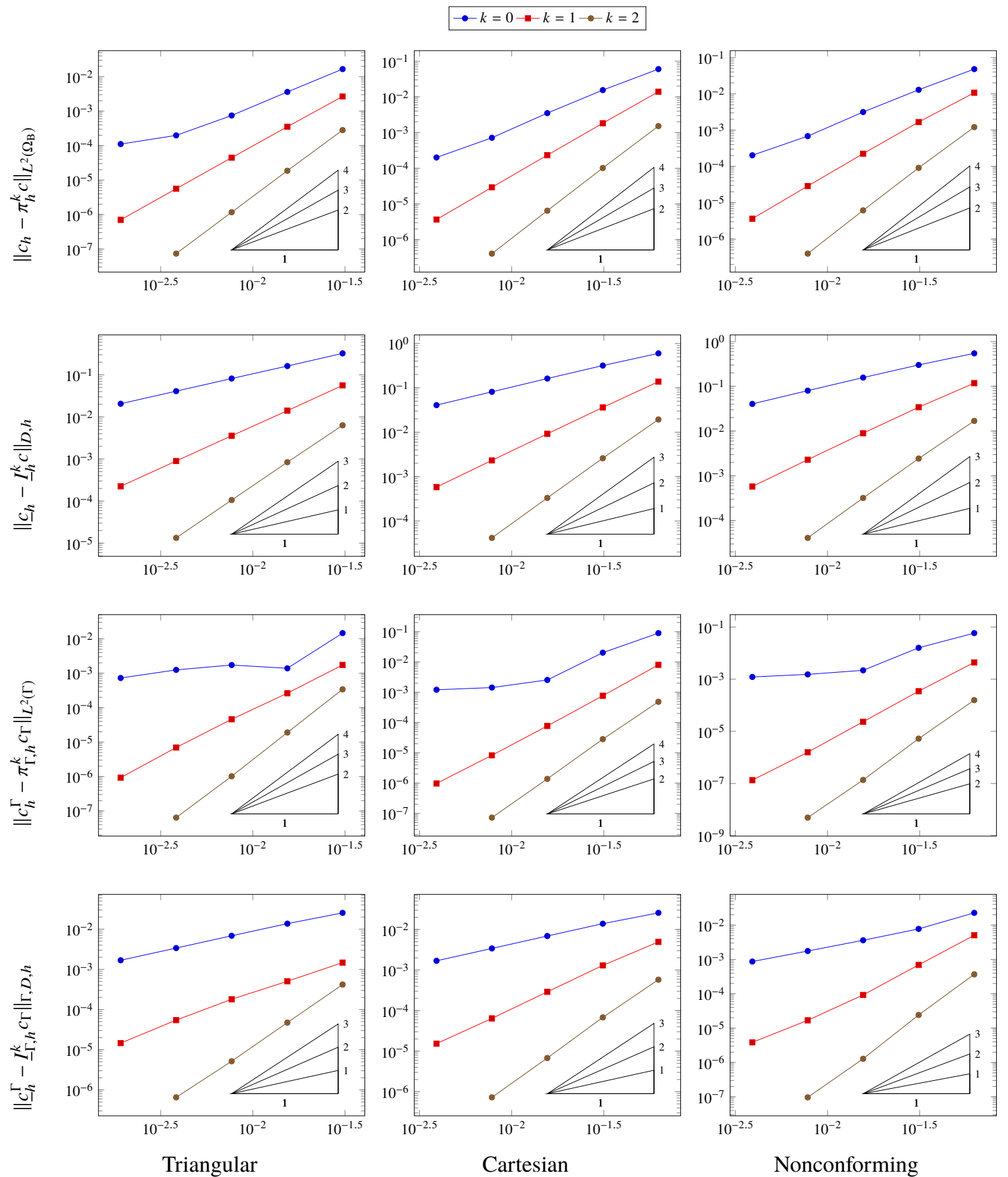

Figure 5: Convergence results for the test case of Section 5.1. Errors (48) for the passive transport problem v. $h$ on the triangular, Cartesian and nonconforming mesh families of Figure 3. 

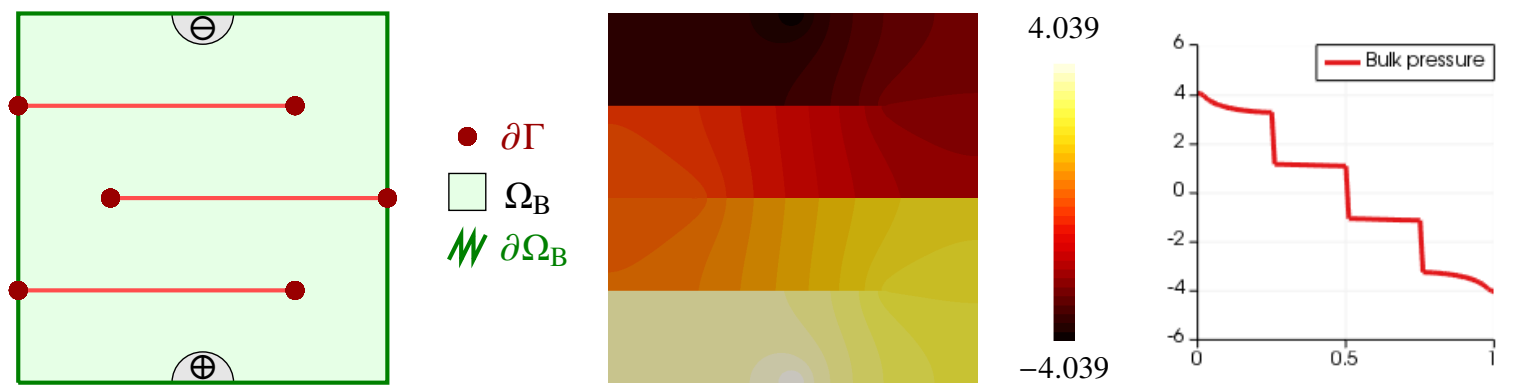

(a) Domain configuration (left), bulk pressure $p$ (middle) and bulk pressure profile over $x_{1}=0.5$ (right).

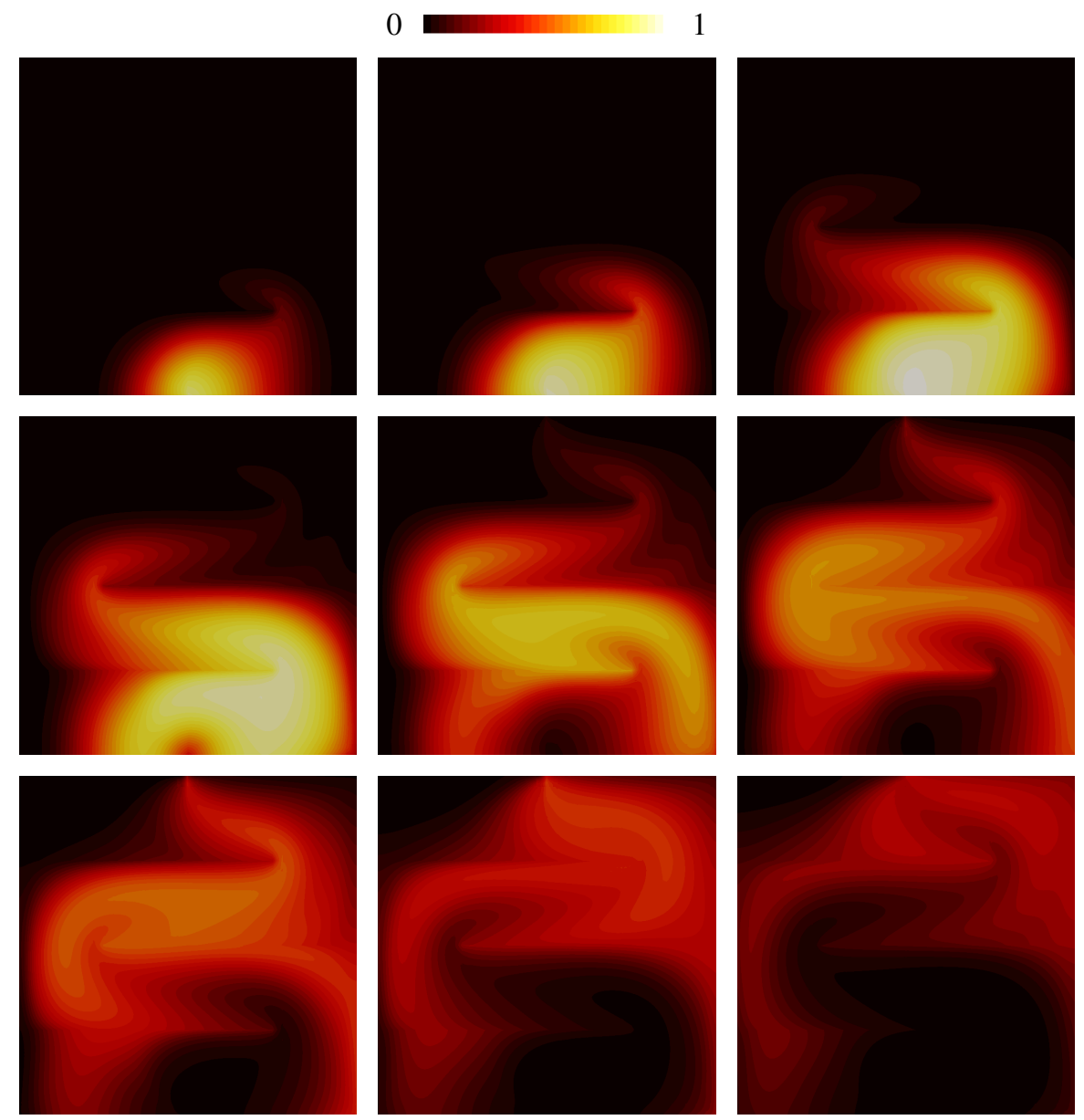

(b) Snapshots of the bulk concentration $c$ at times (from left to right, top to bottom): $t=$ 5, 10, 20, 30, 40, 50, 60, 80, 100.

Figure 6: Configuration and numerical results for the test of Section 5.2 (unsteady transport with impermeable fractures). 


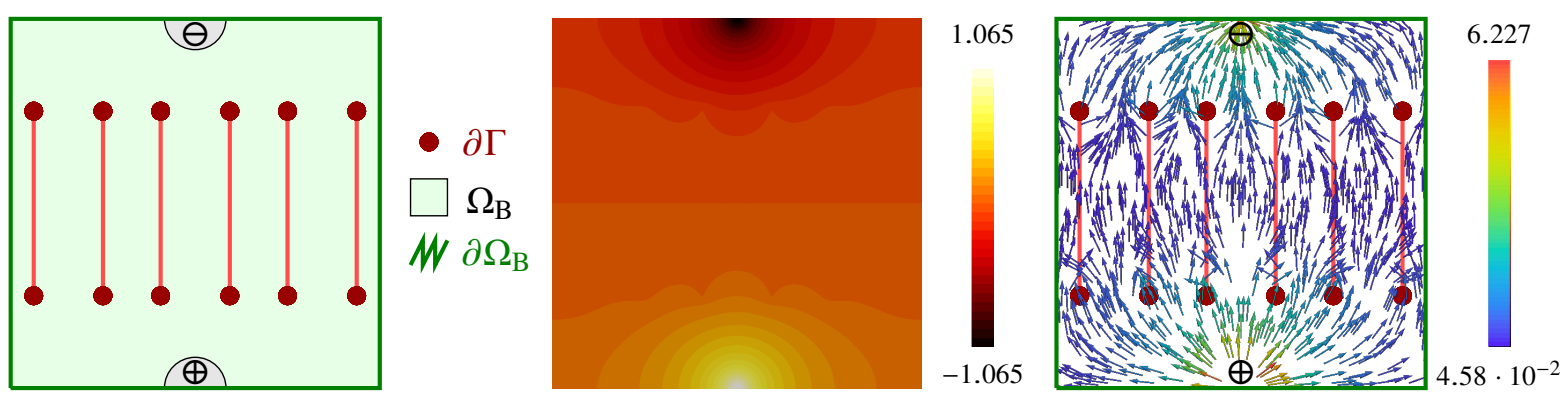

(a) Domain configuration (left), bulk pressure $p$ (middle), and Darcy velocity $\boldsymbol{u}$ (right).
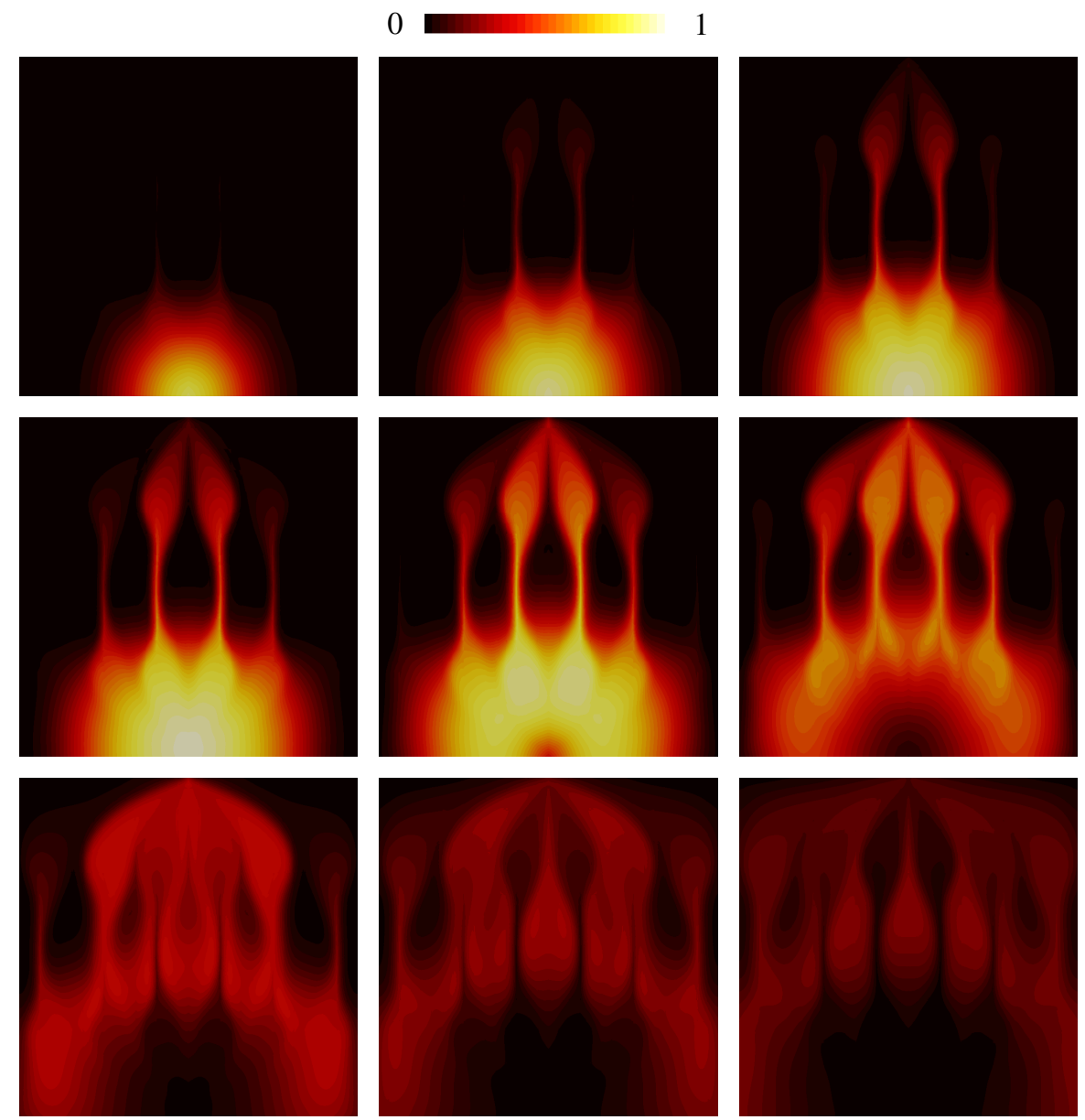

(b) Snapshots of the bulk concentration $c$ at times (from left to right, top to bottom): $t=$ 5, 10, 15, 20, 30, 40, 60, 80, 100.

Figure 7: Configuration and numerical results for the test of Section 5.3 (unsteady transport with permeable fractures). 